This is the final peer-reviewed accepted manuscript of:

Lanza, A., Morigi, S. \& Sgallari, F. Convex Image Denoising via Non-convex Regularization with Parameter Selection. J Math Imaging Vis 56, 195-220 (2016).

The final published version is available online at: https://doi.org/10.1007/s10851016-0655-7

Rights / License:

The terms and conditions for the reuse of this version of the manuscript are specified in the publishing policy. For all terms of use and more information see the publisher's website.

This item was downloaded from IRIS Università di Bologna (https://cris.unibo.it/)

When citing, please refer to the published version. 


\title{
CONVEX IMAGE DENOISING VIA NON-CONVEX REGULARIZATION WITH PARAMETER SELECTION
}

\author{
ALESSANDRO LANZA*, SERENA MORIGI ${ }^{\dagger}$, AND FIORELLA SGALLARI ${ }^{\ddagger}$
}

\begin{abstract}
We introduce a Convex Non-Convex (CNC) denoising variational model for restoring images corrupted by Additive White Gaussian Noise (AWGN). We propose the use of parameterized non-convex regularizers to effectively induce sparsity of the gradient magnitudes in the solution, while maintaining strict convexity of the total cost functional. Some widely used non-convex regularization functions are evaluated and a new one is analyzed which allows for better restorations. An efficient minimization algorithm based on the Alternating Directions Methods of Multipliers (ADMM) strategy is proposed for simultaneously restoring the image and automatically selecting the regularization parameter by exploiting the discrepancy principle. Theoretical convexity conditions for both the proposed CNC variational model and the optimization sub-problems arising in the ADMM-based procedure are provided which guarantee convergence to a unique global minimizer. Numerical examples are presented which indicate how the proposed approach is particularly effective and well suited for images characterized by moderately sparse gradients.
\end{abstract}

Key words. image denoising; variational methods; non-convex non-smooth regularization; alternating directions method of multipliers; discrepancy principle; additive white gaussian noise.

1. Introduction. Noise is an unavoidable component of digital image acquisition, and as such, noise removal is a fundamental task in digital image processing. The challenge is to preserve and enhance important features during the denoising process. The most commonly studied noise model is AWGN, where the observed noisy image $b \in \mathbb{R}^{n}$ is related to the underlying true image $u \in \mathbb{R}^{n}$ by the degradation model

$$
b=u+\eta,
$$

where images are represented in vectorized row-major form and $\eta$ is supposed to be at each point in space independently and identically distributed as a zero-mean Gaussian random variable.

In this paper, we focus on the restoration of images characterized by sparse gradients, feature which, in general, characterizes piecewise constant images.

The denoised image $u$ is computed as the solution of the minimization problem

$$
\min _{u \in \mathbb{R}^{n}}\left\{\mathcal{J}(u)=\sum_{i=1}^{n} \phi\left(\left\|(\nabla u)_{i}\right\|_{2} ; a\right)+\frac{\mu}{2}\|u-b\|_{2}^{2}\right\}
$$

where $\mu>0$ is the so-called regularization parameter, $\phi: \mathbb{R} \rightarrow \mathbb{R}$ is the regularization (or penalty) function parameterized by variable $a$, and $(\nabla u)_{i} \in \mathbb{R}^{2}$ denotes the discretization of the gradient of image $u$ at pixel $i$, so that: $\left\|(\nabla u)_{i}\right\|_{2}=\sqrt{\left(D_{h} u\right)_{i}^{2}+\left(D_{v} u\right)_{i}^{2}}$, with the linear operators $D_{h}, D_{v} \in \mathbb{R}^{n \times n}$ representing finite difference approximations of first-order horizontal and vertical partial derivatives, respectively. The first term in (1.2) is commonly referred to as the regularization term and the second term as

* Department of Mathematics, University of Bologna, Bologna, Italy. Email: alessandro.lanza2@unibo.it.

$\dagger$ Department of Mathematics, University of Bologna, Bologna, Italy. Email: serena.morigi@unibo.it.

$\ddagger$ Department of Mathematics, University of Bologna, Bologna, Italy. Email: fiorella.sgallari@unibo.it. 
the fidelity term. The purpose of the regularization parameter $\mu$ is to balance the influence of the fidelity and regularization terms on the computed solution in a suitable manner.

The purpose of the regularization term in (1.2) is to encode a prior on the image gradients magnitude, and the function $\phi$ is intended so as to strongly promote sparsity and consequently to better fit gradient distributions of real images.

A very popular choice for $\phi$ is the Tikhonov regularization, which involves a smooth, convex regularization term, i.e. $\ell_{2}$ norm, $\phi(t)=t^{2}$. Many methods have been proposed, see for example [12],[4],[5], which allow for effective image restorations in case of smooth images. However, it is well known that Tikhonov regularization does not well preserve edges in the restoration process. Non-smooth, convex regularization terms, e.g. Total Variation (TV) regularization [26], i.e. $\ell_{1}$ norm, $\phi(t)=|t|$, better allow for discontinuities in the restored image, making them an acceptable penalty function for images. Convex formulations benefit from convex optimization theory which leads to robust algorithms with guaranteed convergence. On the other hand, non-smooth, non-convex regularization has remarkable advantages over convex regularization for restoring images, in particular to restore high-quality piecewise constant images with neat edges [16],[22]. However, it may lead to challenging computation since it requires non-convex non-smooth minimization which, involving many minima, can often get stuck in shallow local minima. The non-smooth non-convex and non-Lipschitz regularization term, $\ell_{p}$ quasi-norm, $\phi(t)=|t|^{p}$, with $0<p<1$, has recently been proposed in image processing and compressed sensing since it promotes gradient-sparser solutions or sparser solutions, substantially improving upon the $\ell_{1}$ norm results [29].

In (1.2) the proposed functional $\mathcal{J}(u)$ is given by the sum of a convex smooth (quadratic) fidelity term and a non-convex non-smooth regularization term. The quadratic fidelity term is strongly convex and its positive second-order derivatives holds the potential for compensating the negative second-order derivatives in the regularization term. Hence, $\mathcal{J}(u)$ is surely non-smooth but can be convex or nonconvex depending on the parameter $a$, strictly related to $\mu$. We employ penalty functions parameterized by variable $a$, i.e., $\phi(t)=\phi(t ; a)$, where $a$ is selected so as to ensure convexity of the total cost function $\mathcal{J}(u)$. Consequently, the minimizer is unique and can be computed by using efficient convex optimization algorithms.

The idea of constructing and then optimizing convex functionals containing nonconvex (sparsity-promoting) regularization terms, referred to as Convex-NonConvex (CNC) strategy, was first introduced by Blake and Zisserman in [2], then proposed by Nikolova in [18] for the denoising of binary images and very recently by Selesnik and others for different purposes, see [27], [7], [28], [23], [15] for more details. The attractiveness of such $\mathrm{CNC}$ approach resides in its ability to promote sparsity more strongly than it is possible by using convex regularization while at the same time maintaining the convexity of the optimization problem, so that well-known reliable convex minimization approaches can be used to compute the (unique) solution.

Concerning the regularization parameter $\mu$, it plays a crucial role in the variational model (1.2), and it needs to be suitably set in order to yield good results. In practice, usually the choice of such parameter is made empirically with trial and error; this implies (1.2) to be solved repeatedly for many different values of the parameter $\mu$. Assuming that an estimate $\hat{\epsilon}$ of the norm of the error $\eta$ in the vector $b$ in (1.1) is available, a more efficient strategy for the automatic selection of $\mu$ is based on the 
discrepancy principle [12]. A vector $u_{\mu}$ such that

$$
\left\|u_{\mu}-b\right\|_{2} \leq \bar{\delta}:=\tau_{d} \hat{\epsilon}, \quad \hat{\epsilon} \approx\|\eta\|_{2},
$$

holds, is said to satisfy the discrepancy principle, where $\tau_{d}$ is a user-specified constant independent of $\hat{\epsilon}$ and related to the estimation accuracy of it. Strategies for parameter selection in TV-based image restoration using the discrepancy principle have been proposed e.g. in [30] and [14]. In this paper we generalize these strategies for the proposed CNC model.

Taking into account both the convexity aspect and the discrepancy principle, we propose the following variational denoising model:

$$
\min _{u \in \mathbb{R}^{n}} \mathcal{J}(u ; \mu, a), \quad \mathcal{J}(u ; \mu, a):=\sum_{i=1}^{n} \phi\left(\left\|(\nabla u)_{i}\right\|_{2} ; a\right)+\frac{\mu}{2}\|u-b\|_{2}^{2},
$$

where the parameters pair $(\mu, a) \in \mathbb{R}_{+}^{*} \times \mathbb{R}_{+}$is such that (1.3) is satisfied and $\mathcal{J}(u ; \mu, a)$ is strictly convex in $u$.

The paper is organized as follows. In Section 2 we formally define the class of penalty functions $\phi$ to be used in the proposed model (1.4) and give some popular examples. We also introduce a novel exponential penalty function. In Section 3 we derive sufficient conditions on the parameters pair $(\mu, a)$ for the objective functional $\mathcal{J}$ in (1.4) to be strictly convex. An efficient ADMM-based iterative algorithm for the solution of (1.4) is presented in Section 4, where we also provide an additional condition for convexity of the ADMM sub-problems. Numerical experiments are presented in Section 5, where the proposed convex model with non-convex non-smooth regularization is compared with a convex regularized convex model and a non-convex regularized non-convex model. Conclusions are drawn in Section 6.

2. Non-convex penalty functions. In this section we formally define the class of non-convex penalty functions $\phi$ used in the regularization term of the considered model (1.4). In the following, we denote the sets of non-negative and positive real numbers as $\mathbb{R}_{+}:=\{t \in \mathbb{R}: t \geq 0\}$ and $\mathbb{R}_{+}^{*}:=\{t \in \mathbb{R}: t>0\}$, respectively. We consider parameterized penalty functions $\phi(t ; a): \mathbb{R}_{+} \rightarrow \mathbb{R}$ such that for any value of the parameter $a \in \mathbb{R}_{+}^{*}$ the following assumptions are satisfied:
A1) $\begin{cases}\phi(t ; a) & \in \mathcal{C}^{2}\left(\mathbb{R}_{+}^{*}\right) \\ \phi(t ; a) & \in \mathcal{C}^{0}\left(\mathbb{R}_{+}\right)\end{cases}$
( $\phi$ twice continuously differentiable in $t$ on $\mathbb{R}_{+}^{*}$ )
( $\phi$ continuous in $t$ on $\mathbb{R}_{+}$)
A2) $\quad \phi^{\prime}(t ; a)>0 \quad \forall t \in \mathbb{R}_{+}^{*}$
( $\phi$ strictly increasing in $t$ on $\mathbb{R}_{+}^{*}$ )
A3) $\quad \phi^{\prime \prime}(t ; a) \leq 0 \quad \forall t \in \mathbb{R}_{+}^{*}$
( $\phi$ concave in $t$ on $\mathbb{R}_{+}^{*}$ )
*A4) $\left\{\begin{array}{l}\sup _{t \in \mathbb{R}_{+}^{*}} \phi^{\prime}(t ; a)<+\infty \\ \inf _{t \in \mathbb{R}_{+}^{*}} \phi^{\prime \prime}(t ; a)>-\infty\end{array}\right.$
( $\phi^{\prime}$ bounded from above)
( $\phi^{\prime \prime}$ bounded from below)

Assumptions A1)-A3) are quite standard and encompass a wide class of non-convex sparsity-promoting penalty functions. Assumption *A4), which corresponds to imposing boundedness (from above/below) of first- and second-order derivatives of the penalty function $\phi$, is mandatory when constructing CNC functionals (see, e.g., [27], [7], [28], [23], [15]). In fact, if the second-order derivative of the penalty function goes to $-\infty$ at any point in the domain, there are no chances to compensate it by the positive but bounded second-order derivatives of the convex quadratic fidelity 
term. In particular, we notice that the popular $\ell_{p}$ quasi-norm with $0<p<1$, namely $\phi(t ; p)=t^{p}$, satisfies assumptions A1)-A3) but not assumption A4), since $\sup _{t \in \mathbb{R}_{+}^{*}} \phi^{\prime}(t ; p)=+\infty, \inf _{t \in \mathbb{R}_{+}^{*}} \phi^{\prime \prime}(t ; p)=-\infty$. As a consequence, such penalty function does not allow for applying the CNC strategy.

We notice that, without loss of generality, assumption *A4) can be replaced with the following

A4) $\phi(0 ; a)=0, \sup _{t \in \mathbb{R}_{+}^{*}} \phi^{\prime}(t ; a)=1, \inf _{t \in \mathbb{R}_{+}^{*}} \phi^{\prime \prime}(t ; a)=-a \quad\left(\phi, \phi^{\prime}, \phi^{\prime \prime}\right.$ normalization).

as demonstrated in the Proposition 2.1 below, where, at the same time, the procedure for normalizing the penalty functions is given.

The above assumption A4) calls for a useful normalization of the penalty functions which has been used, e.g., in [27], [7], [15]. By imposing that $\inf _{t \in \mathbb{R}_{+}} \phi^{\prime \prime}(t ; a)=-a$ yields the parameter $a$ to represent a scalar indicator of the "degree of concavity" of the penalty function $\phi$, thus justifying the name concavity parameter.

Finally, we notice that any penalty function satisfying assumptions A1)-A4) tends to the identity function $f(t)=t, t \geq 0$, as the concavity parameter $a$ approaches zero from the right (in fact, $\lim _{a \searrow 0} \phi^{\prime \prime}(t ; a)=0 \forall t \in \mathbb{R}_{+}^{*}$ ). Hence, we complete the class of penalty functions $\phi(t ; a)$ defined by A1)-A4) for $a \in \mathbb{R}_{+}^{*}$ by imposing the following:

$$
\phi(t ; a):=t \text { for } a=0
$$

According to $(2.1)$, the $\ell_{1}$-norm penalty is recovered as a special case of the parameterized penalty function $\phi(\cdot ; a)$ when $a=0$.

Proposition 2.1. Let $\varphi(\cdot ; a)$ be a function satisfying assumptions A1)-*A4) and let $\kappa_{0}, \kappa_{1}, \kappa_{2}: \mathbb{R}_{+}^{*} \rightarrow \mathbb{R}$ be the functions of the concavity parameter a defined by

$$
\kappa_{0}(a):=\varphi(0 ; a), \quad \kappa_{1}(a):=\sup _{t \in \mathbb{R}_{+}^{*}}\left[\varphi^{\prime}(t ; a)\right], \quad \kappa_{2}(a):=\inf _{t \in \mathbb{R}_{+}^{*}}\left[\varphi^{\prime \prime}(t ; a)\right] .
$$

Then, the function $\phi(\cdot ; a)$ defined by

$$
\phi(t ; a):=\frac{\varphi(\alpha(a) t ; a)-\kappa_{0}(a)}{\alpha(a) \kappa_{1}(a)}, \quad \text { with } \quad \alpha(a):=-a \frac{\kappa_{1}(a)}{\kappa_{2}(a)}
$$

satisfies assumptions A1)-A3) and A4).

Proof. First we notice that, according to assumptions A2) and A3) for $\varphi$, we have that $\kappa_{1}(a)>0$ and $\kappa_{2}(a)<0$ for any $a>0$. Hence, in $(2.3)$ we have $\alpha(a)>0$ and $\alpha(a) \kappa_{1}(a)>0$, so that $\phi$ is always defined. By substituting $t=0$ in (2.3) and recalling the definition of $\kappa_{0}(a)$ in $(2.2)$, we obtain:

$$
\phi(0 ; a)=\frac{\varphi(0 ; a)-\kappa_{0}(a)}{\alpha(a) \kappa_{1}(a)}=\frac{\kappa_{0}(a)-\kappa_{0}(a)}{\alpha(a) \kappa_{1}(a)}=0 .
$$

The first- and second-order derivatives with respect to $t$ of function $\phi$ in $(2.3)$ are as follows:

$$
\phi^{\prime}(t ; a)=\frac{\varphi^{\prime}(\alpha(a) t ; a)}{\kappa_{1}(a)}, \quad \phi^{\prime \prime}(t ; a)=-a \frac{\varphi^{\prime \prime}(\alpha(a) t ; a)}{\kappa_{2}(a)} .
$$


Since, in general, for any given function $f: \mathbb{R} \rightarrow \mathbb{R}$ and any given scalars $c_{1}, c_{2}>0$, it holds that inf $/ \sup _{t>0}\left[c_{1} f\left(c_{2} t\right)\right]=c_{1} \inf / \sup _{t>0}[f(t)]$, we have:

$$
\begin{aligned}
& \sup _{t>0}\left[\phi^{\prime}(t ; a)\right]=\quad \frac{\sup _{t>0}\left[\varphi^{\prime}(t ; a)\right]}{\kappa_{1}(a)}=\frac{\kappa_{1}(a)}{\kappa_{1}(a)}=1, \\
& \inf _{t>0}\left[\phi^{\prime \prime}(t ; a)\right]=-a \frac{\inf _{t>0}\left[\varphi^{\prime \prime}(t ; a)\right]}{\kappa_{2}(a)}=-a \frac{\kappa_{2}(a)}{\kappa_{2}(a)}=-a,
\end{aligned}
$$

thus completing the proof.

In the first three columns of Table 2.1 we report three widely used examples of (sparsity-promoting) penalty functions, referred to as $\phi_{\log }, \phi_{\text {rat }}$ and $\phi_{\text {atan }}$, which satisfy all the above assumptions A1)-A4) and have been considered, e.g., in [27], [7], [15]. In particular, the penalty $\phi_{\text {atan }}$ has been proposed in [27] as the maximally sparsity-inducing function among those characterized by a first-order derivative of inverse quadratic polynomial type. For this function, the first-order derivative approaches zero more rapidly than any other function in its class.

We propose a novel penalty function of exponential type, referred to as $\phi_{\text {exp }}$. The expressions of $\phi_{\exp }$ and of its derivatives are reported in the last column of Table 2.1. Since the first-order derivative of $\phi_{\exp }$ approaches to zero as an inverse exponential, that is more rapidly than the other three considered penalties, the $\phi_{\exp }$ penalty allows for inducing sparsity more strongly.

In Figure 2.1 we show the plots of the penalty functions $\phi_{\log }, \phi_{\text {rat }}, \phi_{\text {atan }}, \phi_{\exp }$ and of their first- and second-order derivatives for two different values $a \in\{2,3\}$ of the concavity parameter. It can be noticed from Figures 2.1(a-b) how the proposed penalty $\phi_{\exp }$ approaches its asymptotic value more rapidly than all the other penalties. In fact, as illustrated in Figures 2.1(c-d), the first-order derivative of $\phi_{\exp }$ tends to zero more quickly than all the other penalties. Equivalently, the shape of $\phi_{\exp }$ is more similar to the sparsity-promoting $\ell_{0}$ quasi-norm penalty function.

The proposed penalty function $\phi_{\exp }$ allows for an efficient closed-form threshold function which will be derived in Section 4 .

3. Convexity Conditions. In this section, we investigate convexity of the proposed functional $\mathcal{J}(\cdot ; \mu ; a)$ in (1.4). More precisely, we seek for conditions on the regularization parameter $\mu \in \mathbb{R}_{+}^{*}$ and on the concavity parameter $a \in \mathbb{R}_{+}$of the non-convex penalty functions $\phi(\cdot ; a)$ such that the functional is strictly convex.

Towards this aim, we rewrite $\mathcal{J}(\cdot ; \mu ; a)$ in (1.4) in explicit double-indexed form:

$$
\begin{aligned}
\mathcal{J}(u ; \mu, a) & =\sum_{(i, j) \in \Omega} \phi\left(\frac{1}{\Delta} \sqrt{\left(u_{i+1, j}-u_{i, j}\right)^{2}+\left(u_{i, j+1}-u_{i, j}\right)^{2}} ; a\right) \\
& +\sum_{(i, j) \in \Omega} \frac{\mu}{2}\left(u_{i, j}-b_{i, j}\right)^{2}
\end{aligned}
$$

where $\Omega$ represents the (square) image lattice defined as

$$
\Omega:=\left\{(i, j) \in \mathbb{Z}^{2}: i=1, \ldots, \sqrt{n}, j=1, \ldots, \sqrt{n}\right\},
$$




\begin{tabular}{|r||c|c|c|c|}
\hline & $\phi_{\log }$ & $\phi_{\text {rat }}$ & $\phi_{\text {atan }}$ & $\phi_{\text {exp }}$ \\
\hline \hline$\phi(t ; a)$ & $\frac{\log (1+a t)}{a}$ & $\frac{t}{1+a t / 2}$ & $\frac{\operatorname{atan}\left(\frac{1+2 a t}{\sqrt{3}}\right)-\frac{\pi}{6}}{a \sqrt{3} / 2}$ & $\frac{1-e^{-a t}}{a}$ \\
\hline$\phi^{\prime}(t ; a)$ & $\frac{1}{1+a t}$ & $\frac{1}{(1+a t / 2)^{2}}$ & $\frac{1}{1+a t+a^{2} t^{2}}$ & $\frac{1}{e^{a t}}$ \\
\hline$\phi^{\prime \prime}(t ; a)$ & $-\frac{a}{(1+a t)^{2}}$ & $-\frac{a}{(1+a t / 2)^{3}}$ & $-\frac{a(1+2 a t)}{\left(1+a t+a^{2} t^{2}\right)^{2}}$ & $-\frac{a}{e^{a t}}$ \\
\hline$\phi^{\prime \prime \prime}(t ; a)$ & $\frac{2 a^{2}}{(1+a t)^{3}}$ & $\frac{3 a^{2}}{2(1+a t / 2)^{4}}$ & $\frac{6 a^{3} t(1+a t)}{\left(1+a t+a^{2} t^{2}\right)^{3}}$ & $\frac{a^{2}}{e^{a t}}$ \\
\hline$\phi(0 ; a)$ & 0 & 0 & 0 & 0 \\
\hline $\sup _{t>0} \phi^{\prime}(t ; a)$ & 1 & 1 & $-a$ & 1 \\
\hline $\inf _{t>0} \phi^{\prime \prime}(t ; a)$ & $-a$ & $-a$ & $\frac{2}{a} \frac{\pi \sqrt{3}}{9}$ & $\frac{1}{a}$ \\
\hline $\lim _{t \rightarrow+\infty} \phi(t ; a)$ & $+\infty$ & $\frac{2}{a}$ & & 0 \\
\hline
\end{tabular}

TABLE 2.1

Examples of penalty functions $\phi(t ; a): \mathbb{R}_{+} \rightarrow \mathbb{R}_{+}$satisfying assumptions $\left.\left.A 1\right)-A_{4}\right)$.

and where a standard first-order forward finite difference scheme has been used for discretization of the first-order partial derivatives, with $\Delta=\Delta_{x}=\Delta_{y}$ denoting the discretization step size. We notice that the convexity results obtained at the end of this section depend on the chosen finite difference scheme. Nevertheless, the procedure used below for deriving such results can be adapted for inferring the convexity conditions associated with other discretization choices.

To the purpose of splitting (3.1) into the sum of easier functions, we define the sets of odd and even integers

$$
\mathrm{O}:=\{i \in \mathbb{Z}: i \text { is odd }\} \quad \text { and } \quad \mathrm{E}:=\{i \in \mathbb{Z}: i \text { is even }\}
$$

and we introduce the following partition of $\Omega$, illustrated in Figure 3.1(left):

$$
\begin{array}{ll}
\mathrm{OO}:=\{(i, j) \in \Omega: i \in \mathrm{O}, j \in \mathrm{O}\} & \mathrm{EE}:=\{(i, j) \in \Omega: i \in \mathrm{E}, j \in \mathrm{E}\} \\
\mathrm{OE}:=\{(i, j) \in \Omega: i \in \mathrm{O}, j \in \mathrm{E}\} & \mathrm{EO}:=\{(i, j) \in \Omega: i \in \mathrm{E}, j \in \mathrm{O}\}
\end{array}
$$

It is easy to show that our functional in (3.1) can be split into the following sum:

$$
\mathcal{J}(u ; \mu, a)=\mathcal{J}_{\mathrm{OO}}(u ; \mu, a)+\mathcal{J}_{\mathrm{EE}}(u ; \mu, a)+\mathcal{J}_{\mathrm{OE}}(u ; \mu, a)+\mathcal{J}_{\mathrm{EO}}(u ; \mu, a),
$$

where

$$
\begin{aligned}
& \mathcal{J}_{A}(u ; \mu, a)=\sum_{(i, j) \in A} \phi\left(\frac{1}{\Delta} \sqrt{\left(u_{i+1, j}-u_{i, j}\right)^{2}+\left(u_{i, j+1}-u_{i, j}\right)^{2}} ; a\right) \\
& +\sum_{(i, j) \in A}\left[\frac{\mu}{6}\left(u_{i, j}-b_{i, j}\right)^{2}+\frac{\mu}{6}\left(u_{i+1, j}-b_{i+1, j}\right)^{2}+\frac{\mu}{6}\left(u_{i, j+1}-b_{i, j+1}\right)^{2}\right],
\end{aligned}
$$




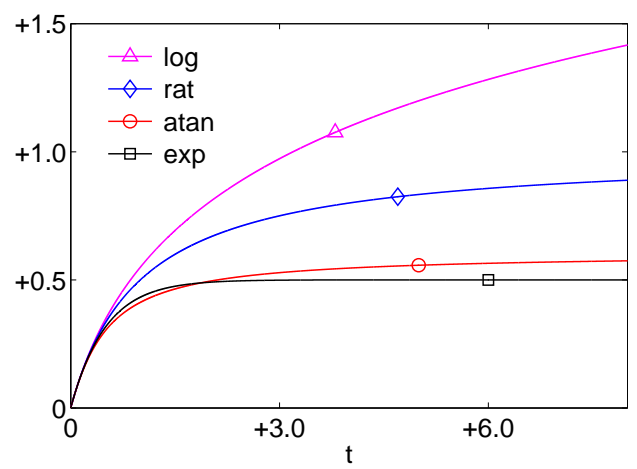

(a) $\phi(t ; a), a=2$



(c) $\phi^{\prime}(t ; a), a=2$



(e) $\phi^{\prime \prime}(t ; a), a=2$

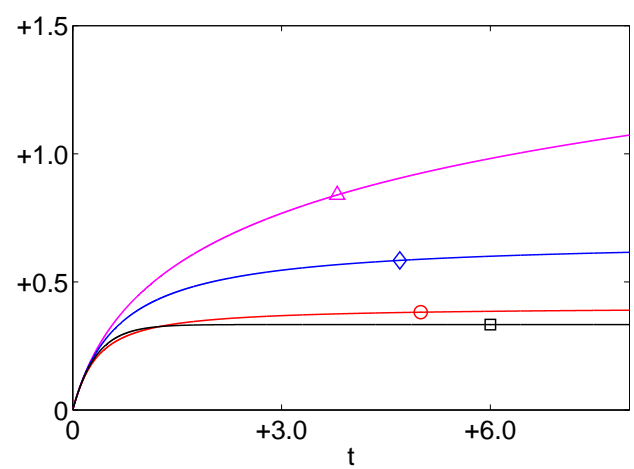

(b) $\phi(t ; a), a=3$

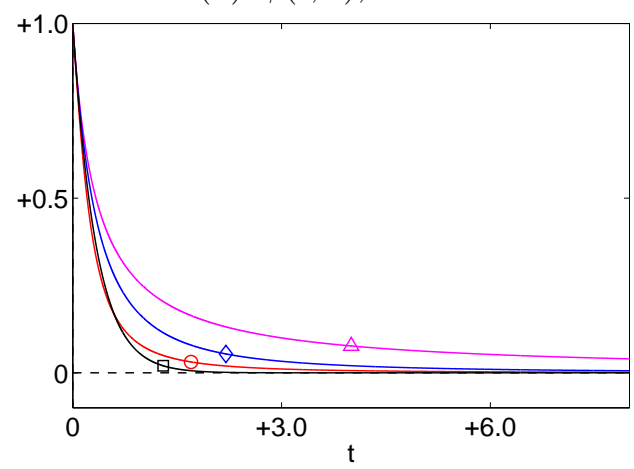

(d) $\phi^{\prime}(t ; a), a=3$

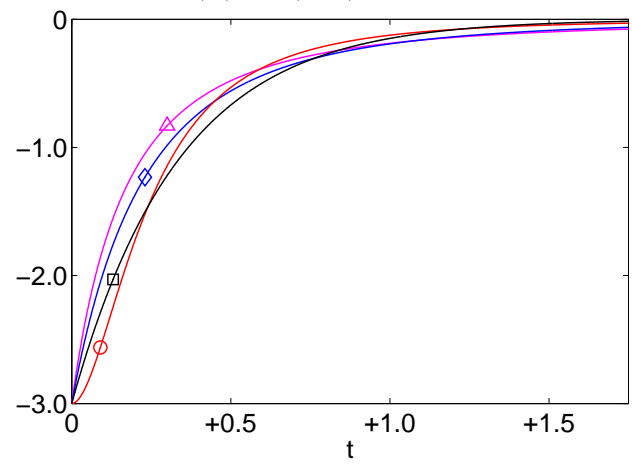

(f) $\phi^{\prime \prime}(t ; a), a=3$

FIG. 2.1. Plots of the penalty functions $\phi_{\log }(t ; a), \phi_{\operatorname{rat}}(t ; a), \phi_{\operatorname{atan}}(t ; a), \phi_{\exp }(t ; a)$ defined in Table 2.1 and of their first-and second-order derivatives for different values of the concavity parameter a.

with $A \in\{O O, E E, O E, E O\}$. Since the four functions $\mathcal{J}_{\mathrm{OO}}, \mathcal{J}_{\mathrm{EE}}, \mathcal{J}_{\mathrm{OE}}$ and $\mathcal{J}_{\mathrm{EO}}$ defined in (3.6) have exactly the same structure, convexity of $\mathcal{J}$ can be analyzed by just considering the function $\mathcal{J}_{\text {OO }}$. In Figure 3.1(right) we illustrate the subset of the image lattice $\Omega$ involved by function $\mathcal{J}_{\mathrm{OO}}$.

Let us define the function $f(\cdot ; \mu, a): \mathbb{R}^{3} \rightarrow \mathbb{R}$ as

$$
f\left(x_{1}, x_{2}, x_{3} ; \mu, a\right)=\frac{\mu}{6}\left(x_{1}^{2}+x_{2}^{2}+x_{3}^{2}\right)+\phi\left(\frac{1}{\Delta} \sqrt{\left(x_{2}-x_{1}\right)^{2}+\left(x_{3}-x_{1}\right)^{2}} ; a\right) .
$$



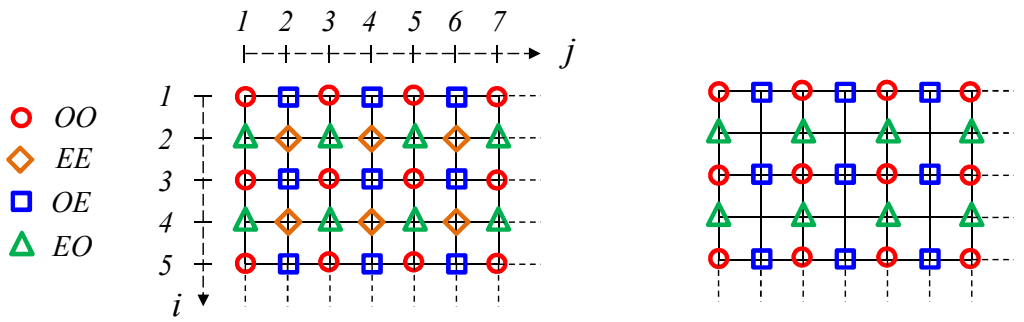

FIG. 3.1. Partition of the image lattice $\Omega$ in (3.2) into the four subsets $O O, E E, O E$ and $E O$ defined in (3.4) (left); image stencil involved by function $\mathcal{J}_{\mathrm{OO}}$ (right).

Then, from (3.6) the function $\mathcal{J}_{\mathrm{OO}}$ can be rewritten as follows:

$$
\mathcal{J}_{\mathrm{OO}}(u)=\sum_{(i, j) \in \mathrm{OO}}\left[f\left(u_{i, j}, u_{i+1, j}, u_{i, j+1}\right)+L\left(u_{i, j}, u_{i+1, j}, u_{i, j+1}\right)\right],
$$

where $L$ is an affine function which, hence, does not affect convexity of $\mathcal{J}_{\text {OO }}$.

To study convexity of functional $\mathcal{J}(\cdot ; \mu, a)$ defined in (1.4), we give three propositions which allow to reduce convexity investigation from the original functional $\mathcal{J}(\cdot ; \mu, a)$ of $n$ variables to easier functions $f(\cdot ; \mu, a), g(\cdot ; \mu, a)$ and $h(\cdot ; \mu, a)$ of three, two and one variables, respectively.

Proposition 3.1. The functional $\mathcal{J}(\cdot ; \mu, a)$ defined in (1.4) is strictly convex if the function $f(\cdot ; \mu, a): \mathbb{R}^{3} \rightarrow \mathbb{R}$ defined in (3.7) is strictly convex.

The proof is straightforward given (3.5)-(3.8) and the the above discussion.

Proposition 3.2. The function $f(\cdot ; \mu, a): \mathbb{R}^{3} \rightarrow \mathbb{R}$ defined in (3.7) is strictly convex if and only if the function $g(\cdot ; \mu, a): \mathbb{R}^{2} \rightarrow \mathbb{R}$ is strictly convex, with

$$
g\left(y_{1}, y_{2} ; \mu, a\right)=\frac{\mu}{6}\left(y_{1}^{2}+y_{2}^{2}\right)+\phi\left(\frac{1}{\Delta} \sqrt{y_{1}^{2}+y_{2}^{2}} ; a\right) .
$$

The proof is postponed to the appendix.

Proposition 3.3. The function $g(\cdot ; \mu, a): \mathbb{R}^{2} \rightarrow \mathbb{R}$ defined in (3.9) is strictly convex if and only if the function $h(\cdot ; \mu, a): \mathbb{R}_{+} \rightarrow \mathbb{R}$ defined as

$$
h(t ; \mu, a)=\frac{\mu}{6} t^{2}+\phi\left(\frac{t}{\Delta} ; a\right)
$$

is monotonically increasing and strictly convex.

The proof is postponed to the appendix.

TheOREM 3.4. For any parameters pair $(\mu, a) \in \mathbb{R}_{+}^{*} \times \mathbb{R}_{+}$and any function $\phi(\cdot ; a): \mathbb{R}_{+} \rightarrow \mathbb{R}_{+}$satisfying assumptions A1)-A4) in Section 2, the functional $\mathcal{J}(\cdot ; \mu, a): \mathbb{R}^{n} \rightarrow \mathbb{R}$ defined in (1.4) is strictly convex if the following condition holds:

$$
a<\frac{\mu}{3} \Delta^{2} .
$$


Proof. Based on Propositions 3.1-3.3, the functional $\mathcal{J}(\cdot ; \mu, a)$ defined in (1.4) is strictly convex if the twice differentiable function $h(\cdot ; \mu, a)$ defined in (3.10) is monotonically increasing and strictly convex everywhere in its domain $\mathbb{R}_{+}$, that is if the following two conditions hold:

$$
\left\{\begin{array} { l } 
{ h ^ { \prime } ( t ; \mu , a ) = \frac { \mu } { 3 } t + \frac { 1 } { \Delta } \phi ^ { \prime } ( \frac { t } { \Delta } ; a ) > 0 } \\
{ h ^ { \prime \prime } ( t ; \mu , a ) = \frac { \mu } { 3 } + \frac { 1 } { \Delta ^ { 2 } } \phi ^ { \prime \prime } ( \frac { t } { \Delta } ; a ) > 0 }
\end{array} \equiv \left\{\begin{array}{l}
\phi^{\prime}\left(\frac{t}{\Delta} ; a\right)>-\frac{\mu}{3} \Delta t \\
\phi^{\prime \prime}\left(\frac{t}{\Delta} ; a\right)>-\frac{\mu}{3} \Delta^{2}
\end{array} \forall t \geq 0 .\right.\right.
$$

Since by hypothesis $\mu>0, \Delta>0$ and for any $a \geq 0$ the penalty function $\phi(\cdot ; a)$ is monotonically increasing in $\mathbb{R}_{+}$, the first condition in (3.12) is always satisfied. Recalling assumption A4) in Section 2, the second condition in (3.12) can be equivalently rewritten as in (3.11).

We introduce a scalar coefficient $\tau_{c}$, referred to as the convexity coefficient, aimed at setting the degree of convexity of the functional $\mathcal{J}$, and we rewrite convexity condition (3.11) in the following equivalent form:

$$
a=\tau_{c} \frac{\mu}{3} \Delta^{2}, \quad \tau_{c} \in[0,1[.
$$

In particular, we notice that for $\tau_{c}$ approaching 1 from the left the penalty function $\phi$ is maximally concave thus allowing for more strongly promoting sparsity.

We conclude this section by formalizing the main properties of functional $\mathcal{J}$ in (1.4).

Proposition 3.5. For any parameters pair ( $\mu, a)$ satisfying condition (3.13), the functional $\mathcal{J}(u ; \mu, a)$ in (1.4) is proper, continuous (hence, lower semi-continuous), coercive and strictly convex.

Proof. Functional $\mathcal{J}$ in (1.4) is clearly proper. Moreover, as the quadratic fidelity term and the functions $\phi$ and $\|\cdot\|_{2}$ in the regularization term are continuous, $\mathcal{J}$ is continuous. We notice that the regularization term in $\mathcal{J}$ is coercive if and only if the penalty function $\phi$ is coercive, which is not always the case (see the last row of Table 2.1). However, $\mathcal{J}$ is coercive due to the strongly convex quadratic fidelity term. Finally, $\mathcal{J}$ is strictly convex due to Theorem 3.4 .

4. Applying ADMM to the proposed CNC model. In this section, we illustrate in detail the ADMM-based [3] iterative algorithm used to numerically solve the proposed CNC model (1.4). Towards this aim, first we resort to the variable splitting technique [1] and introduce two auxiliary variables $z \in V$ and $t \in Q$, with $V:=\mathbb{R}^{n}$, $Q:=\mathbb{R}^{2 n}$. In particular, the variable $t$ is defined such that $t_{i}:=\left(\left(D_{h} u\right)_{i},\left(D_{v} u\right)_{i}\right) \in \mathbb{R}^{2}$ represents the discrete gradient of image $u$ at pixel $i$. After defining the matrix $D:=\left(D_{h}^{T}, D_{v}^{T}\right)^{T} \in \mathbb{R}^{2 n \times n}$, model (1.4) is thus rewritten in the following equivalent form:

$$
\begin{gathered}
\left\{u^{*}, z^{*}, t^{*}\right\} \leftarrow \arg \min _{u, z, t}\left\{\sum_{i=1}^{n} \phi\left(\left\|t_{i}\right\|_{2} ; a\right)+\frac{\mu}{2}\|z-b\|_{2}^{2}\right\} \\
\text { subject to : } z=u, \quad t=D u,
\end{gathered}
$$

where $\mu$ and $a$ are such that (1.3) and (3.13) are satisfied.

We remark that the auxiliary variable $t$ is introduced to transfer the discrete gradient operators $(\nabla u)_{i}$ in (1.4) out of the non-convex non-smooth regularization 
term $\phi\left(\|\cdot\|_{2} ; a\right)$. The variable $z$ plays the role of the restored image $u$ within the discrepancy principle-based constraint (1.3), so that the constraint is now imposed on $z$ instead of $u$.

To solve problem (4.1), we define the augmented Lagrangian functional

$$
\begin{aligned}
\mathcal{L}\left(u, z, t ; \lambda_{z}, \lambda_{t} ; \mu, a\right)= & \sum_{i=1}^{n} \phi\left(\left\|t_{i}\right\|_{2} ; a\right)+\frac{\mu}{2}\|z-b\|_{2}^{2} \\
& -\left\langle\lambda_{z}, z-u\right\rangle+\frac{\beta_{z}}{2}\|z-u\|_{2}^{2} \\
& -\left\langle\lambda_{t}, t-D u\right\rangle+\frac{\beta_{t}}{2}\|t-D u\|_{2}^{2},
\end{aligned}
$$

where $\beta_{z}, \beta_{t}>0$ are scalar penalty parameters and $\lambda_{z} \in V, \lambda_{t} \in Q$ are the vectors of Lagrange multipliers associated with the linear constraints $z=u$ and $t=D u$ in (4.2), respectively. We then consider the following saddle-point problem:

$$
\begin{aligned}
\text { Find } & \left(u^{*}, z^{*}, t^{*} ; \lambda_{z}^{*}, \lambda_{t}^{*}\right) \in V \times V \times Q \times V \times Q \\
\text { s.t. } & \mathcal{L}\left(u^{*}, z^{*}, t^{*} ; \lambda_{z}, \lambda_{t} ; \mu, a\right) \leq \mathcal{L}\left(u^{*}, z^{*}, t^{*} ; \lambda_{z}^{*}, \lambda_{t}^{*} ; \mu, a\right) \leq \mathcal{L}\left(u, z, t ; \lambda_{z}^{*}, \lambda_{t}^{*} ; \mu, a\right) \\
& \forall\left(u, z, t ; \lambda_{z}, \lambda_{t}\right) \in V \times V \times Q \times V \times Q,
\end{aligned}
$$

with the augmented Lagrangian functional $\mathcal{L}$ defined in (4.3).

In the following we prove that, for any parameters pair $(\mu, a)$ satisfying (convexity) condition (3.13), the saddle-point problem (4.3)-(4.4) has at least one solution and, more importantly, all its solutions will provide the unique minimizer of the strictly convex functional $\mathcal{J}(u ; \mu, a)$ in (1.4). Analogous proofs have been previously given for the case of TV regularization with quadratic [14] and also with convex non-quadratic [31] fidelity terms. However, in our case the regularization term, that for simplicity we denote in the following by

$$
\Phi(u ; a):=\sum_{i=1}^{n} \phi\left(\left\|(\nabla u)_{i}\right\|_{2} ; a\right),
$$

is a non-convex generalization of the TV which calls for a suitable adaptation of the above mentioned proofs based on concepts from calculus for non-smooth non-convex functions, namely the generalized (or Clarke) gradients [9].

In the following we will denote by $\partial_{x}[f]\left(x^{*}\right)$ and by $\bar{\partial}_{x}[f]\left(x^{*}\right)$ the subdifferential (in the sense of convex analysis [24],[25]) and the Clarke generalized gradient [9], respectively, with respect to $x$ of the function $f$ calculated at $x^{*}$.

In Lemma 4.1 below we give some results on locally Lipschitz continuity for the functions involved in the subsequent demonstrations, which are necessary for the generalized gradients being defined. Then, in Proposition 4.2 we give first-order optimality conditions for our functional $\mathcal{J}(u ; \mu, a)$. These conditions, together with results in Lemma 4.3 proved in [11], will be crucial for demonstrating the equivalence (in terms of solutions) between the minimization problem in (1.4) and the saddle-point problem in (4.3)-(4.4), as done in the final Theorem 4.4.

Lemma 4.1. For any parameters pair $(\mu, a)$ satisfying (convexity) condition (3.13), the functional $\mathcal{J}$ in (1.4) and, separately, the $\Phi$ regularization term in (4.5) and the quadratic fidelity term, are locally Lipschitz continuous functions. 
Proof. The proof is immediate by considering that $\mathcal{J}$ and the quadratic fidelity term are convex functions, hence locally Lipschitz, and that the $\Phi$ term in (4.5) is composition of locally Lipschitz functions (note that the penalty functions $\phi(\cdot ; a)$ defined in Section 2 are globally 1-Lipschitz for any $a \in \mathbb{R}_{+}$).

Proposition 4.2. For any parameters pair ( $\mu, a)$ satisfying (convexity) condition (3.13), the functional $\mathcal{J}: \mathbb{R}^{n} \rightarrow \mathbb{R}$ in (1.4) has a unique minimizer $u^{*}$ which satisfies

$$
0 \in \partial_{u}[\mathcal{J}]\left(u^{*}\right)
$$

where 0 denotes the null vector in $\mathbb{R}^{n}$ and $\partial_{u}[\mathcal{J}]\left(u^{*}\right) \subset \mathbb{R}^{n}$ represents the subdifferential (with respect to $u$, calculated at $u^{*}$ ) of functional $\mathcal{J}$. Moreover, it follows that

$$
0 \in D^{T} \bar{\partial}_{D u}[\Phi]\left(D u^{*}\right)+\mu\left(u^{*}-b\right),
$$

where $\bar{\partial}_{D u}[\Phi]\left(D u^{*}\right) \subset \mathbb{R}^{2 n}$ denotes the Clarke generalized gradient (with respect to $D u$, calculated at $\left.D u^{*}\right)$ of the non-convex non-smooth regularization term $\Phi$ in (4.5).

The proof is postponed to the appendix.

Lemma 4.3. [11] Assume that $F=F_{1}+F_{2}, F_{1}$ and $F_{2}$ being lower semicontinuous convex functions of $\mathbb{R}^{n}$ into $\mathbb{R}, F_{1}$ being Gâteaux-differentiable with differential $F_{1}^{\prime}$. Then, if $p^{*} \in \mathbb{R}^{n}$, the following two conditions are equivalent to each other:

1. $p^{*}$ is a solution of $\inf _{p} F(p)$;

2. $\left\langle F_{1}^{\prime}\left(p^{*}\right), p-p^{*}\right\rangle+F_{2}(p)-F_{2}\left(p^{*}\right) \geq 0 \quad \forall p \in \mathbb{R}^{n}$.

THEOREM 4.4. For any parameters pair $(\mu, a)$ satisfying (convexity) condition (3.13), the saddle-point problem (4.3)-(4.4) admits at least one solution and all the solutions have the form $\left(u^{*}, u^{*}, D u^{*} ; \lambda_{z}^{*}, \lambda_{t}^{*}\right)$, with $u^{*}$ denoting the unique global minimizer of functional $\mathcal{J}(u ; \mu, a)$ in (1.4).

The proof is postponed to the appendix.

In the following we present the ADMM-based iterative algorithm used to compute a saddle-point solution of (4.3)-(4.4) which, according to Theorem 4.4, provides the unique global minimizer of functional $\mathcal{J}(u ; \mu, a)$ in $(1.4)$.

Given the previously computed (or initialized for $k=0$ ) vectors $u^{(k)}, \lambda_{z}^{(k)}$ and $\lambda_{t}^{(k)}$, the $k$-th iteration of the proposed ADMM-based iterative scheme applied to the solution of the saddle-point problem (4.3)-(4.4) reads as follows:

$$
\begin{aligned}
& z^{(k+1)} \leftarrow \arg \min _{z \in V} \mathcal{L}\left(u^{(k)}, z, t^{(k)} ; \lambda_{z}^{(k)}, \lambda_{t}^{(k)}\right) \\
& t^{(k+1)} \leftarrow \arg \min _{t \in Q} \mathcal{L}\left(u^{(k)}, z^{(k+1)}, t ; \lambda_{z}^{(k)}, \lambda_{t}^{(k)}\right) \\
& u^{(k+1)} \leftarrow \arg \min _{u \in V} \mathcal{L}\left(u, z^{(k+1)}, t^{(k+1)} ; \lambda_{z}^{(k)}, \lambda_{t}^{(k)}\right) \\
& \lambda_{z}^{(k+1)} \leftarrow \lambda_{z}^{(k)}-\gamma \beta_{z}\left(z^{(k+1)}-u^{(k+1)}\right) \\
& \lambda_{t}^{(k+1)} \leftarrow \lambda_{t}^{(k)}-\gamma \beta_{t}\left(t^{(k+1)}-D u^{(k+1)}\right) \\
& 11
\end{aligned}
$$


where $\gamma>0$ is a relaxation parameter. In the following we show in detail how to solve the three minimization sub-problems (4.8)-(4.10) for the primal variables $z, t$ and $u$, respectively, then we present the overall iterative ADMM-based minimization algorithm.

Computing $\boldsymbol{z}^{(\boldsymbol{k}+1)}$ (and setting $\left.\boldsymbol{\mu}^{(\boldsymbol{k}+1)}\right)$. Given $u^{(k)}$ and $\lambda_{z}^{(k)}$, and recalling the definition of the augmented Lagrangian functional in (4.3), the minimization subproblem for $z$ in (4.8) can be rewritten as follows:

$$
\begin{aligned}
z^{(k+1)} & \leftarrow \arg \min _{z \in V}\left\{\frac{\mu}{2}\|z-b\|_{2}^{2}+\frac{\beta_{z}}{2}\left\|z-u^{(k)}\right\|_{2}^{2}-\left\langle\lambda_{z}^{(k)}, z-u^{(k)}\right\rangle\right\} \\
& \leftarrow \arg \min _{z \in V}\left\{\mu\|z-b\|_{2}^{2}+\beta_{z}\left\|z-q^{(k+1)}\right\|_{2}^{2}\right\}
\end{aligned}
$$

with the constant (w.r.t. the optimization variable $z$ ) vector $q^{(k+1)} \in V$ given by:

$$
q^{(k+1)}=u^{(k)}+\frac{1}{\beta_{z}} \lambda_{z}^{(k)} .
$$

The cost function in (4.13) is strictly convex in $z$ and its (unique) global minimizer is as follows:

$$
z_{\mu}^{(k+1)}=\frac{\mu b+\beta_{z} q^{(k+1)}}{\mu+\beta_{z}},
$$

where the dependency of the minimizer on the regularization parameter $\mu$ is made explicit. We notice that, following from (4.15), the minimizer $z_{\mu}^{(k+1)}$ belongs to the line segment between $b$ and $q^{(k+1)}$. In particular, we have that $z_{\mu=0}^{(k+1)}=q^{(k+1)}$ and $z_{\mu \rightarrow+\infty}^{(k+1)}=b$.

We define the discrepancy associated with the minimizer $z_{\mu}^{(k+1)}$ in (4.15) as a function $\delta^{(k+1)}: \mathbb{R}_{+} \rightarrow \mathbb{R}_{+}$of the regularization parameter $\mu$ defined as follows:

$$
\delta^{(k+1)}(\mu):=\left\|z_{\mu}^{(k+1)}-b\right\|_{2}=\frac{\beta_{z}}{\left(\mu+\beta_{z}\right)}\left\|q^{(k+1)}-b\right\|_{2},
$$

where the second equality comes from substituting the explicit expression of $z_{\mu}^{(k+1)}$ given in (4.15). Recalling that $\beta_{z}>0$, the discrepancy function in (4.16) has the following properties:

$$
\begin{aligned}
\delta^{(k+1)} \in \mathcal{C}^{\infty}\left(\mathbb{R}_{+}\right), & \delta^{(k+1)}(\mu)>0,\left(\delta^{(k+1)}\right)^{\prime}(\mu)<0 \quad \forall \mu \in \mathbb{R}_{+}, \\
\delta^{(k+1)}(0)=\left\|q^{(k+1)}-b\right\|_{2}, & \delta^{(k+1)}(+\infty)=0 .
\end{aligned}
$$

In order to choose a suitable value $\mu^{(k+1)}$ of the regularization parameter $\mu$ such that the discrepancy principle in (1.3) is satisfied here for the auxiliary variable $z$, we consider two cases.

In case that $\left\|q^{(k+1)}-b\right\|_{2} \leq \bar{\delta}$, then we have:

$$
\delta^{(k+1)}(\mu) \leq \frac{\beta_{z}}{\left(\mu+\beta_{z}\right)} \bar{\delta} \leq \bar{\delta} \quad \forall \mu \in \mathbb{R}_{+},
$$

that is the discrepancy principle is satisfied for any possible $\mu$. We choose $\mu^{(k+1)}=0$, such that, according to (4.15), the solution of (4.13) is $z^{(k+1)}=q^{(k+1)}$. 
In case that $\left\|q^{(k+1)}-b\right\|_{2}>\bar{\delta}$, the discrepancy function properties in (4.17) guarantee that there exists a unique value $\mu^{(k+1)}$ of the regularization parameter $\mu$ such that $\delta^{(k+1)}\left(\mu^{(k+1)}\right)=\bar{\delta}$ (in fact, the discrepancy function is monotonically decreasing). Recalling (4.16), we have:

$$
\frac{\beta_{z}}{\left(\mu^{(k+1)}+\beta_{z}\right)}\left\|q^{(k+1)}-b\right\|_{2}=\bar{\delta} \Longleftrightarrow \mu^{(k+1)}=\beta_{z}\left(\frac{\left\|q^{(k+1)}-b\right\|_{2}}{\bar{\delta}}-1\right) .
$$

Replacing $\mu^{(k+1)}$ given in (4.19) for $\mu$ in (4.15), we obtain that in this second case the solution of (4.13) is as follows:

$$
z^{(k+1)}=b+\bar{\delta} \frac{q^{(k+1)}-b}{\left\|q^{(k+1)}-b\right\|_{2}} .
$$

Summarizing, we have:

$$
\mu^{(k+1)}= \begin{cases}0 & \text { if }\left\|q^{(k+1)}-b\right\|_{2} \leq \bar{\delta} \\ \beta_{z}\left(\frac{\left\|q^{(k+1)}-b\right\|_{2}}{\bar{\delta}}-1\right) & \text { if }\left\|q^{(k+1)}-b\right\|_{2}>\bar{\delta}\end{cases}
$$

and consequently:

$$
z^{(k+1)}= \begin{cases}q^{(k+1)} & \text { if } \quad\left\|q^{(k+1)}-b\right\|_{2} \leq \bar{\delta} \\ b+\bar{\delta} \frac{q^{(k+1)}-b}{\left\|q^{(k+1)}-b\right\|_{2}} & \text { if } \quad\left\|q^{(k+1)}-b\right\|_{2}>\bar{\delta}\end{cases}
$$

Computing $t^{(k+1)}$ (and setting $a^{(k+1)}, \beta_{t}^{(k+1)}$ ). Given the new value of the regularization parameter $\mu^{(k+1)}$ computed by (4.21), we set the new value of the concavity parameter $a^{(k+1)}$ such that our functional $\mathcal{J}\left(u ; \mu^{(k+1)}, a^{(k+1)}\right)$ in (1.4) is strictly convex. In particular, according to the convexity conditions in (3.13), we set:

$$
a^{(k+1)}=\tau_{c} \frac{\mu^{(k+1)} \Delta^{2}}{3}, \quad \tau_{c} \in[0,1[,
$$

where $\tau_{c}$ is a user-specified fixed parameter. Then, given $\lambda_{t}^{(k)}$ and $u^{(k)}$, and recalling the definition of the augmented Lagrangian functional in (4.3), the minimization subproblem for $t$ in (4.9) can be rewritten as follows:

$$
\begin{aligned}
t^{(k+1)} & \leftarrow \arg \min _{t \in Q}\left\{\sum_{i=1}^{n} \phi\left(\left\|t_{i}\right\|_{2} ; a^{(k+1)}\right)-\left\langle\lambda_{t}^{(k)}, t-D u^{(k)}\right\rangle+\frac{\beta_{t}}{2}\left\|t-D u^{(k)}\right\|_{2}^{2}\right\} \\
& \leftarrow \arg \min _{t \in Q}\left\{\sum_{i=1}^{n} \phi\left(\left\|t_{i}\right\|_{2} ; a^{(k+1)}\right)+\frac{\beta_{t}}{2}\left\|t-r^{(k+1)}\right\|_{2}^{2}\right\} \\
& \leftarrow \arg \min _{t \in Q} \sum_{i=1}^{n}\left\{\phi\left(\left\|t_{i}\right\|_{2} ; a^{(k+1)}\right)+\frac{\beta_{t}}{2}\left\|t_{i}-r_{i}^{(k+1)}\right\|_{2}^{2}\right\}
\end{aligned}
$$

with the constant (w.r.t. the optimization variable $t$ ) vector $r^{(k+1)} \in Q$ given by:

$$
r^{(k+1)}=D u^{(k)}+\frac{1}{\beta_{t}} \lambda_{t}^{(k)} .
$$


Note that in (4.24) the minimized functional is written in explicit component-wise form, with $r_{i}^{(k+1)}=\left(D u^{(k)}\right)_{i}+\left(\lambda_{t}^{(k)}\right)_{i} / \beta_{t}$ and where $\left(D u^{(k)}\right)_{i},\left(\lambda_{t}^{(k)}\right)_{i} \in \mathbb{R}^{2}$ denote the discrete gradient and the Lagrange multipliers at pixel $i$, respectively. The minimization problem in (4.24) is equivalent to the following $n$ independent 2-dimensional problems:

$$
t_{i}^{(k+1)} \leftarrow \arg \min _{t_{i} \in \mathbb{R}^{2}}\left\{\phi\left(\left\|t_{i}\right\|_{2} ; a^{(k+1)}\right)+\frac{\beta_{t}}{2}\left\|t_{i}-r_{i}^{(k+1)}\right\|_{2}^{2}\right\}, \quad i=1, \ldots, n .
$$

Since by (4.23) we have imposed that the functional $\mathcal{J}\left(u ; \mu^{(k+1)}, a^{(k+1)}\right)$ minimized at iteration $k$ is strictly convex, we clearly aim at avoiding non-convexity of the ADMM sub-problems (4.26). In the first part of Proposition 4.5 below, we give a necessary and sufficient condition for strict convexity of the cost functions in (4.26). In particular, based on (4.31)-(4.32) in Proposition 4.5, we can state that all the problems in (4.26) are strictly convex if and only if the following condition holds:

$$
\beta_{t}>a^{(k+1)} \text {. }
$$

Since we can not further constraint the sequence $\left\{a^{(k)}\right\}$ without damaging its crucial role (together with sequence $\left\{\mu^{(k)}\right\}$ ) in driving the solution towards the convexity and discrepancy constraints, in order to satisfy condition (4.27) we have to act on the ADMM penalty parameter $\beta_{t}$ by thus resorting to an iteration-dependent version of it. In particular, we set:

$$
\beta_{t}^{(k+1)}=\max \left\{\beta_{t}, 1.05 \cdot a^{(k+1)}\right\}
$$

where $\beta_{t}$, similarly to $\beta_{z}$, represents a fixed user-specified parameter.

In case that (4.27) is satisfied, the unique solutions of the strictly convex problems (4.26) can be obtained as reported in the second part of Proposition 4.5, that is:

$$
t_{i}^{(k+1)}=\xi_{i}^{(k+1)} r_{i}^{(k+1)}, \quad i=1, \ldots, n,
$$

where the shrinkage coefficients $\xi_{i}^{(k+1)} \in[0,1[$ are computed according to (4.35), that is:

$$
\xi_{i}^{(k+1)}=\left\{\begin{array}{l}
0 \text { if }\left\|r_{i}^{(k+1)}\right\|_{2} \leq 1 / \beta_{t}^{(k+1)} \\
\left.\xi^{*} \in\right] 0,1\left[\text { s.t. } \phi^{\prime}\left(\left\|r_{i}^{(k+1)}\right\|_{2} \xi^{*} ; a\right)+\beta_{t}^{(k+1)}\left\|r_{i}^{(k+1)}\right\|_{2}\left(\xi^{*}-1\right)=0 \quad\right. \text { otherwise. }
\end{array}\right.
$$

The nonlinear equation in (4.30) can be solved by very few steps of the iterative Newton-Raphson method. In particular, it can be demonstrated that for all the considered penalty functions $\phi_{\log }, \phi_{\text {rat }}, \phi_{\text {atan }}$ and $\phi_{\exp }$ reported in Table 2.1, by choosing $\xi^{*}=1$ as the initial guess, the Newton-Raphson algorithm is guaranteed to converge (with quadratic rate) to the unique root of the nonlinear equation. This is due to the third-order derivative $\phi^{\prime \prime \prime}(\cdot ; a)$ being positive (see Table 2.1$)$.

Proposition 4.5. Let $\beta>0, a \geq 0$ and $r \in \mathbb{R}^{2}$ be given constants, and let $\phi(\cdot ; a): \mathbb{R}_{+} \rightarrow \mathbb{R}_{+}$be a function satisfying assumptions A1)-A4) in Section 2. Then, the function

$$
\theta(x):=\phi\left(\|x\|_{2} ; a\right)+\underset{14}{\frac{\beta}{2}}\|x-r\|_{2}^{2}, \quad x \in \mathbb{R}^{2}
$$


is strictly convex if and only if the following condition holds:

$$
a<\beta .
$$

Moreover, in case that (4.32) holds, the strictly convex minimization problem

$$
\arg \min _{x \in \mathbb{R}^{2}} \theta(x)
$$

admits the unique solution $x^{*}$ given by the following shrinkage operator:

$$
x^{*}=\xi^{*} r, \quad \text { with } \xi^{*} \in[0,1[
$$

equal to
a) $\xi^{*}=0$
if $\|r\|_{2} \leq \frac{1}{\beta}$
b) $\left.\xi^{*} \in\right] 0,1[$ unique solution of :$$
\phi^{\prime}\left(\|r\|_{2} \xi ; a\right)+\beta\|r\|_{2}(\xi-1)=0 \quad \text { otherwise. }
$$

The proof is postponed to the appendix.

For the proposed penalty function $\phi_{\exp }$, the nonlinear equation in (4.30) admits a closed-form solution given in Proposition 4.6 below.

Proposition 4.6. Let $\phi_{\exp }(\cdot ; a): \mathbb{R}_{+} \rightarrow \mathbb{R}_{+}$be the penalty function defined in Table 2.1. Then, the unique solution $\left.\xi^{*} \in\right] 0,1[$ of the nonlinear equation in (4.35) is as follows:

$$
\xi^{*}=1+\frac{1}{a\|r\|_{2}} W_{0}\left(-\frac{a}{\beta e^{a\|r\|_{2}}}\right)
$$

where $W_{0}(\cdot)$ denotes the principal branch of the Lambert $W$ function [10].

The proof is postponed to the appendix.

Computing $\boldsymbol{u}^{(k+1)}$. Given $z^{(k+1)}, t^{(k+1)}, \lambda_{z}^{(k)}$ and $\lambda_{t}^{(k)}$, and recalling the definition of the augmented Lagrangian functional in (4.3), the minimization sub-problem for $u$ in (4.10) can be rewritten as follows:

$$
\begin{aligned}
u^{(k+1)} \leftarrow \arg \min _{u \in V} & \left\{\frac{\beta_{z}}{2}\left\|z^{(k+1)}-u\right\|_{2}^{2}+\frac{\beta_{t}^{(k+1)}}{2}\left\|t^{(k+1)}-D u\right\|_{2}^{2}\right. \\
& \left.+\left\langle\lambda_{z}^{(k)}, u\right\rangle+\left\langle\lambda_{t}^{(k)}, D u\right\rangle\right\},
\end{aligned}
$$

where constant terms have been omitted. The quadratic minimization problem (4.37) has first-order optimality conditions which lead to the following linear system:

$$
\left(\beta_{z} I+\beta_{t}^{(k+1)} D^{T} D\right) u=\beta_{t}^{(k+1)} D^{T}\left(t^{(k+1)}-\frac{1}{\beta_{t}^{(k+1)}} \lambda_{t}^{(k)}\right)+\beta_{z} z^{(k+1)}-\lambda_{z}^{(k)} .
$$


The coefficient matrix of the linear system (4.38) is symmetric positive definite and highly sparse. Hence, (4.38) can be solved very efficiently by the iterative (preconditioned) Conjugate Gradient method. Moreover, under appropriate assumptions about the solution $u$ near the image boundary, the linear system can be solved even more efficiently. For example, under periodic boundary conditions the matrix $D^{T} D$ is block circulant with circulant blocks, so that the coefficient matrix in (4.38) can be diagonalized by the 2D discrete Fourier transform (FFT implementation). However, it is well known that imposing periodic boundary conditions can lead to artifacts in the solution due to artificially introduced image discontinuities. By using more natural reflective or anti-reflective boundary conditions, no artificial discontinuities near the image boundary are introduced, hence higher quality solutions can be obtained. As illustrated in [17] and [8], under reflective and anti-reflective boundary conditions the coefficient matrix in (4.38) can be diagonalized by the $2 \mathrm{D}$ discrete cosine and sine transforms (FCT and FST implementations), respectively. Therefore, at each ADMM iteration the linear system (4.38) can be solved by two forward and one inverse $2 \mathrm{D}$ FFT/FCT/FST, each at a cost of $O(n \log n)$.

ADMM-based minimization algorithm. To summarize previous results, in Algorithm 1 we report the main steps of the ADMM-based iterative scheme used to solve the proposed CNC model (1.4). We point out that by model parameters we mean all the initial choices that define the functional $\mathcal{J}$ in our model (1.4).

For what concern convergence of the ADMM-based algorithm, we point out that the key issues are the usage of two different penalty parameters $\beta_{z}, \beta_{t}$, the variability of the regularization parameter $\mu^{(k)}$ (and, accordingly, of the concavity parameter $\left.a^{(k)}\right)$ during the ADMM iterations, and the non-convexity of the regularization term in our objective functional $\mathcal{J}$ in (1.4). Analysis of convergence of the ADMM subject to the first two issues has been provided in [33], [14]. In particular, the proof in [14] can be adapted to cope with non-convexity of our regularization term.

5. Numerical Examples. In this section, we evaluate experimentally the performance of the proposed CNC variational model (1.4), solved by the ADMM-based scheme in Algorithm 1, when applied to the four $256 \times 256$ test images satellite, phantom, checkboard and qrcode synthetically corrupted by zero-mean AWGN. The uncorrupted test images, shown in Figure 5.1, are mainly characterized by constant regions and sharp edges so as to better highlight the properties of our CNC model.

In the first example we evaluate the performance of the proposed $\mathrm{CNC}$ model when using different penalty functions $\phi(t ; a)$ and different values of the convexity coefficient $\tau_{c}$ and of the discrepancy coefficient $\tau_{d}$. In the second example we investigate the advantages of using convex models with non-convex instead of convex regularization terms, particularly for images characterized by sparse gradients. Finally, in order to evaluate the performance of purely non-convex denoising methods, in the third example we consider the proposed model (1.4) with convexity coefficient $\tau_{c}>1$, that is beyond the limit of convexity for the objective functional $\mathcal{J}$, and we compare it with a state-of-the-art non-convex approach for the denoising of piecewise constant images. We remark that convergence of Algorithm 1 is not theoretically guaranteed when applied to model (1.4) with $\tau_{c}>1$.

More precisely, in the second and third examples we compare the proposed CNC model with the well-known convex Rudin-Osher-Fatemi (ROF) model [26], based on the minimization of the so-called $\mathrm{TV}-\ell_{2}$ functional, with the convex non-local TV (referred to as NLTV) model [32], and with the state-of-the-art non-convex model proposed in [22], whose software is made available by the authors [20]. 


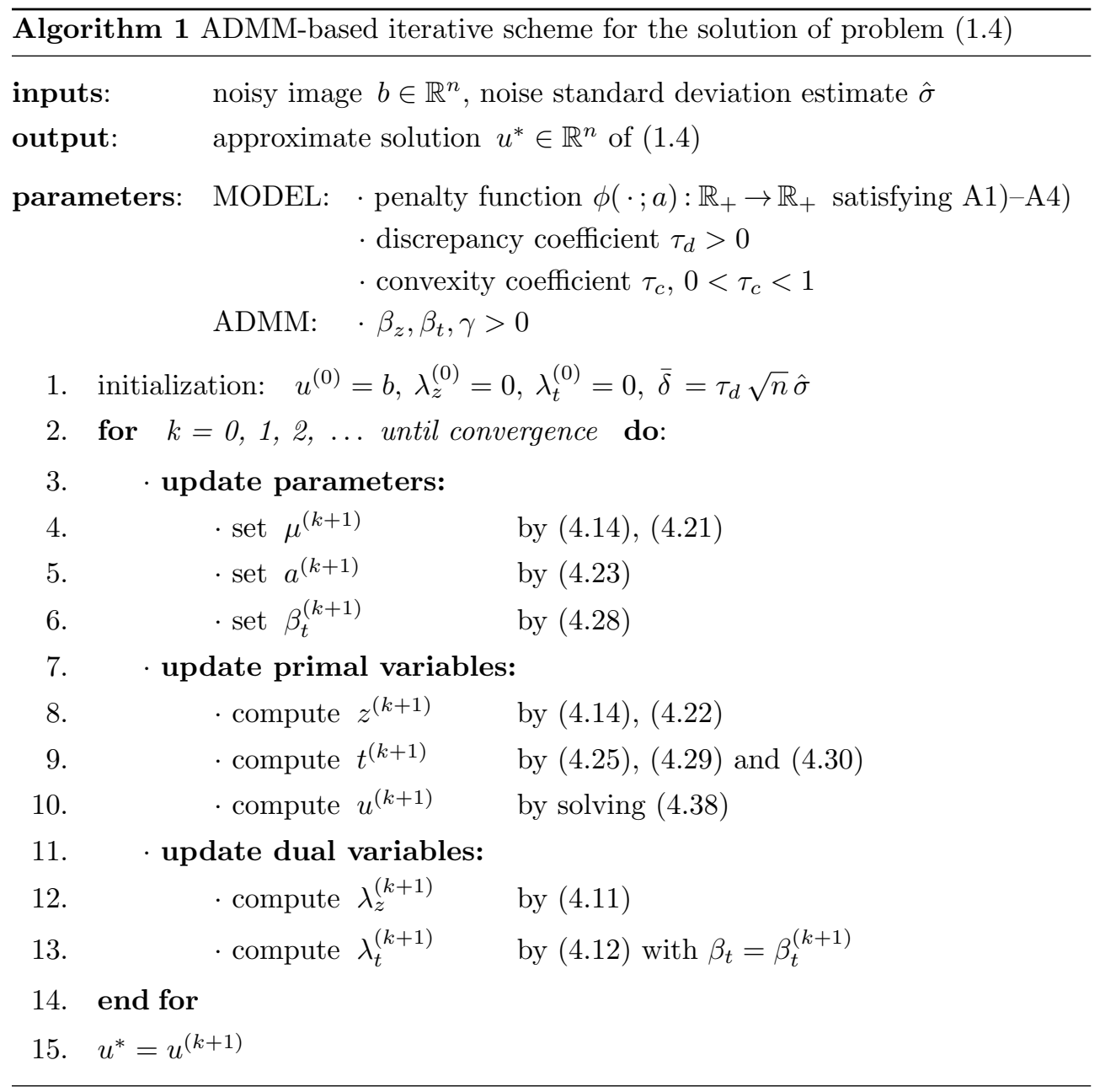

For the minimization of the proposed CNC model we used the ADMM-based procedure reported in Algorithm 1, which requires the relaxation parameter $\gamma$ and the penalty parameters $\beta_{t}$ and $\beta_{z}$ to be set. We used $\gamma=1$ (no relaxation), which is a pretty standard choice, while we set $\beta_{t}=\beta_{z}=50$ such that for all the experiments the condition (4.27) for convexity of the ADMM sub-problem for $t$ is always satisfied. Finally, we set the space step $\Delta=1$, which corresponds to the standard choice of the pixel size.

Since the ROF model is a particular instance of our CNC model (1.4) where $\phi(t)=t$, we used the same ADMM-based scheme as in Algorithm 1 with suitably chosen shrinkage coefficients as described in [6], and with the same parameters $\gamma=1$, $\beta_{t}=\beta_{z}=50$.

In all the experiments and for all the algorithms, we used the observed noisecorrupted image as the initial iterate, i.e. $u^{(0)}=b$, and we terminated the iterations as soon as the relative change between two successive iterates satisfies

$$
\left\|u^{(k)}-u^{(k-1)}\right\|_{2} /\left\|u^{(k-1)}\right\|_{2}<10^{-4} .
$$

The quality of the obtained restorations is evaluated by the Signal-to-Noise Ratio 

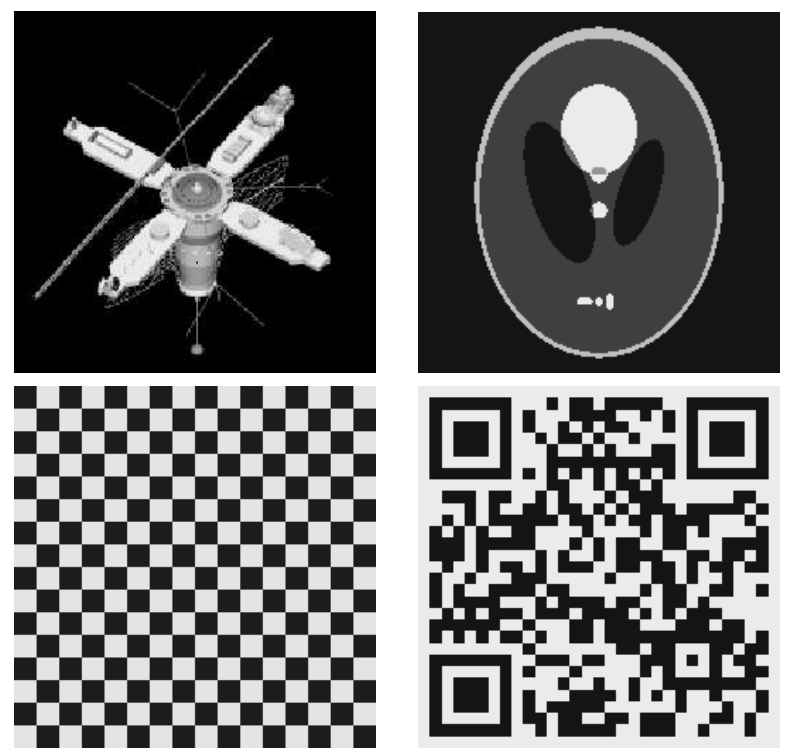

FIG. 5.1. Test images: satellite, phantom, checkboard and qrcode.

(SNR) defined as

$$
\operatorname{SNR}\left(u^{*}, \bar{u}\right):=10 \log _{10}\left(\|\bar{u}-E(\bar{u})\|_{2}^{2} /\left\|u^{*}-\bar{u}\right\|_{2}^{2}\right),
$$

where $u^{*} \in \mathbb{R}^{n}$ is the computed estimate of the uncorrupted image $\bar{u} \in \mathbb{R}^{n}$ and $E(\bar{u})$ denotes the mean value of $\bar{u}$. More precisely, the Improved Signal-to-Noise Ratio (ISNR), defined as

$$
\operatorname{ISNR}\left(u^{*}, \bar{u}, b\right):=\operatorname{SNR}\left(u^{*}, \bar{u}\right)-\operatorname{SNR}(b, \bar{u}),
$$

provides a quantitative measure of the improvement in the quality of the denoised image: a high ISNR value indicates that $u^{*}$ is an accurate approximation of $\bar{u}$.

In all the experiments, the noise-corrupted image $b$ has been synthetically generated by first choosing its associated $\mathrm{SNR}$ value, denoted by $\mathrm{SNR}_{b}$, and then computing the standard deviation $\sigma$ of the zero-mean AWGN corruption yielding such SNR value by the following formula:

$$
\sigma=\frac{\|\bar{u}-E(\bar{u})\|_{2}}{\sqrt{n} 10^{\frac{\mathrm{SNR}_{b}}{20}}} .
$$

The corrupted image $b$ is thus obtained as follows:

$$
b=\bar{u}+\sigma * \operatorname{randn}(\sqrt{n}),
$$

and $\sigma$ is passed as the noise standard deviation estimate to the CNC algorithm.

Example 1. In this example we analyze the performance of the proposed CNC approach when varying the penalty function among those introduced in Table 2.1, the convexity coefficient $\tau_{c}$ in the range $[0,1$ [ such that the minimized functional is convex according to condition (3.13), and the discrepancy coefficient $\tau_{d}$ in a range 


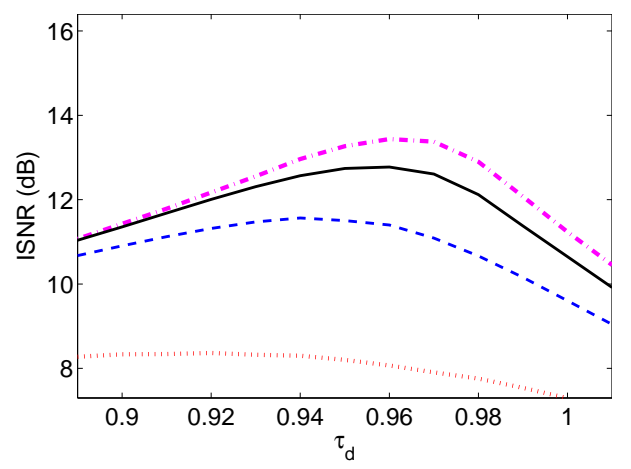

(a) $\phi_{\log }$

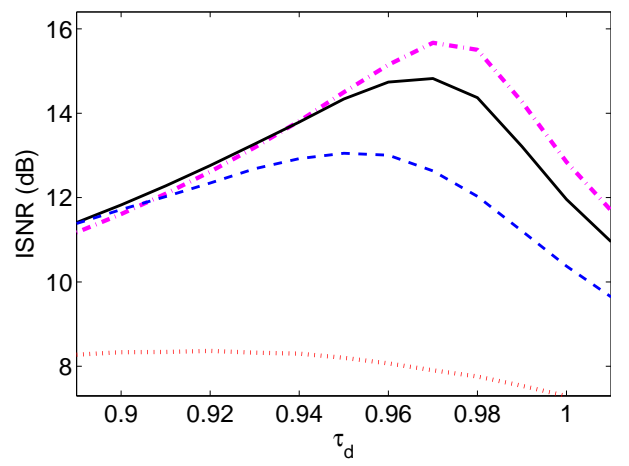

(c) $\phi_{\text {atan }}$

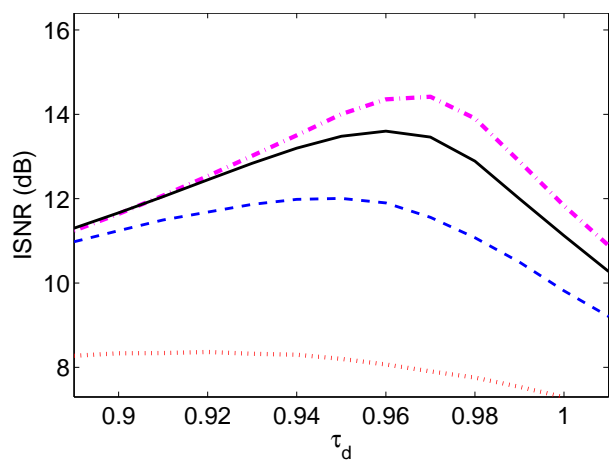

(b) $\phi_{\text {rat }}$

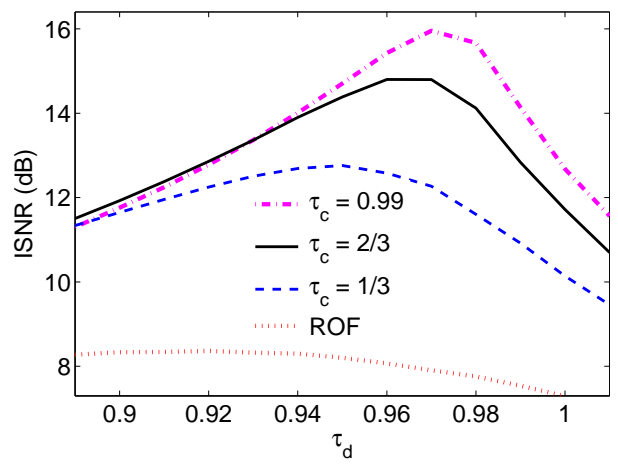

(d) $\phi_{\exp }$

FIG. 5.2. Example 1: Accuracy evaluation (ISNR) of the proposed CNC model in (1.4) with varying penalty function $\phi \in\left\{\phi_{\log }, \phi_{\text {rat }}, \phi_{\text {atan }}, \phi_{\exp }\right\}$, convexity coefficient $\tau_{c} \in\{0,1 / 3,2 / 3,0.99\}$ and discrepancy coefficient $\tau_{d} \in[0.89,1.01]$.

around 1. Evaluation is performed on the test image qrcode shown in Figure 5.1 corrupted by zero-mean AWGN yielding $\mathrm{SNR}_{b}=15$.

The restoration accuracies in terms of ISNR values obtained by using the four considered penalty function $\phi$ are illustrated in the four subplots of Figure 5.2. In each subplot, three curves are associated with three different values of the convexity coefficient $\tau_{c} \in\{1 / 3,2 / 3,0.99\}$ whereas the fourth curve corresponds to the setting (2.1) in Section 2 yielding the penalty function $\phi(t)=t$, i.e. the ROF model.

It is evident from Figure 5.2 that for all the considered penalty functions the quality of the restored images increases with an increasing $\tau_{c}$ until the convexity limit $\left(\tau_{c}=0.99\right)$ is reached. This best choice corresponds to a maximally non-convex regularization preserving the convexity of the total functional $\mathcal{J}$.

Hence, in the next numerical experiment we fix $\tau_{c}=0.99$ and we compare the performance of the different penalty functions in terms of achieved ISNR values as well as in terms of number of performed ADMM iterations, as illustrated in Figures 5.3(left) and 5.3(right), respectively. From the accuracy point of view, the proposed exponential penalty (see Table 2.1) provides the best performance as shown in Figure 5.3 (left), whereas for what concerns the number of required iterations the difference among the penalties is negligible. These observations motivated us to use the penalty function $\phi_{\exp }$ and $\tau_{c}=0.99$ in the remaining numerical experiments. 

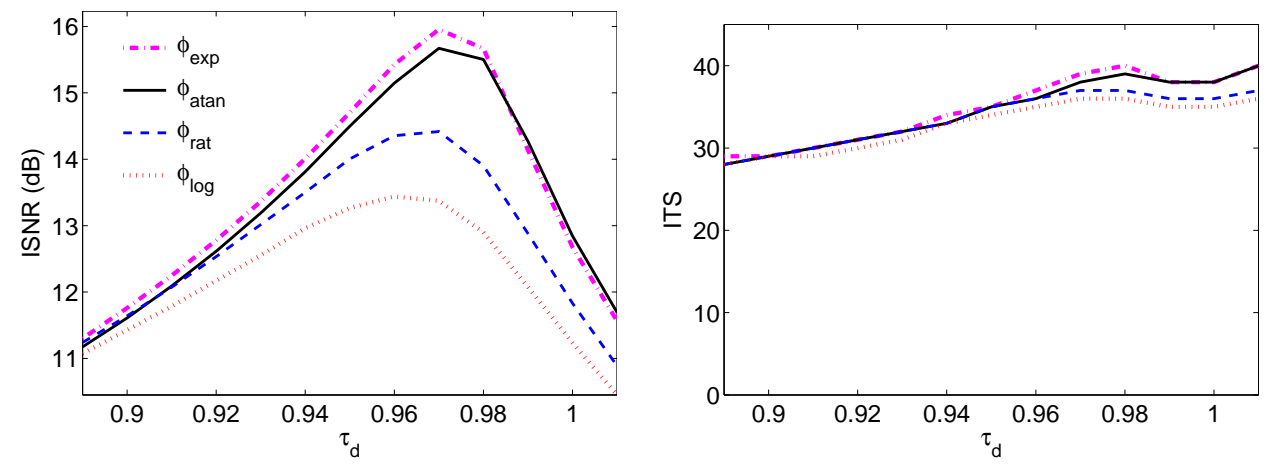

FIG. 5.3. Example 1: Accuracy (ISNR, to the right) and efficiency (iterations, to the left) evaluation of the proposed CNC model in (1.4) with fixed convexity coefficient $\tau_{c}=0.99$ and varying penalty function $\phi \in\left\{\phi_{\log }, \phi_{\mathrm{rat}}, \phi_{\mathrm{atan}}, \phi_{\exp }\right\}$ and discrepancy coefficient $\tau_{d} \in[0.89,1.01]$.

We conclude this example by presenting an empirical investigation on the numerical convergence of the proposed ADMM-based minimization scheme with automatic selection of the regularization parameter reported in Algorithm 1. Towards this aim, in Figure 5.4 we report some convergence plots concerning the case $S N R_{b}=15$ for the qrcode test image. In particular, the first five double-logarithmic plots of Figure 5.4 represent the absolute changes of the three primal variables $u, t, z$ and of the two dual variables $\lambda_{t}, \lambda_{z}$ involved along the iterations $k$ in the ADMM procedure in Algorithm 1. The empirical behavior suggests numerical convergence of the proposed scheme.

In the sixth plot (third row, to the right) of Figure 5.4 we report the ISNR values associated with the iterate $u^{(k)}$, whereas in the fourth row we show the iterates $\mu^{k}$ computed by Algorithm 1 according to the discrepancy principle and the associated discrepancy $\delta^{(k)}$. It can be noticed that both $\mu^{k}$ and $\delta^{(k)}$ converge; in particular, the discrepancy converges towards the prescribed value $\bar{\delta}$, represented by a dashed (red) horizontal line.

Example 2. In this example we carry out a broader evaluation of the proposed CNC model by considering the four test images satellite, phantom, checkboard and qrcode shown in Figure 5.1 corrupted by zero-mean AWGN yielding different $\mathrm{SNR}_{b}$ values. In particular, based on the results presented in the previous example, we use the penalty function $\phi_{\exp }$ and parameter pairs $(\mu, a)$ satisfying the convexity condition (3.13) with convexity coefficient $\tau_{c}=0.99$.

To demonstrate the advantages of using non-smooth non-convex versus nonsmooth but convex regularization in a convex variational model, we compare the performance of our CNC model with the popular ROF or TV- $\ell_{2}$ model [26] and with its non-local extension NLTV- $\ell_{2}$ [32]. In all the experiments, the discrepancy coefficient $\tau_{d}$ for the $\mathrm{TV}-\ell_{2}$ model and for the CNC model has been hand-tuned so as to get the highest possible ISNR value of the denoised image. Similarly, in order to obtain the best possible results for the NLTV- $\ell_{2}$ model we hand-tuned the regularization parameter $\mu$.

In Table 5.1 we report the results of the comparison, with $\mathrm{SNR}_{b}$ denoting the SNR of the noise-corrupted image $b$ and with the best ISNR results marked in boldface. First, we notice that the proposed CNC model outperforms TV- $\ell_{2}$ and NLTV- $\ell_{2}$ in all the examples. In particular, the ISNR improvements obtained by $\mathrm{CNC}$ with respect 

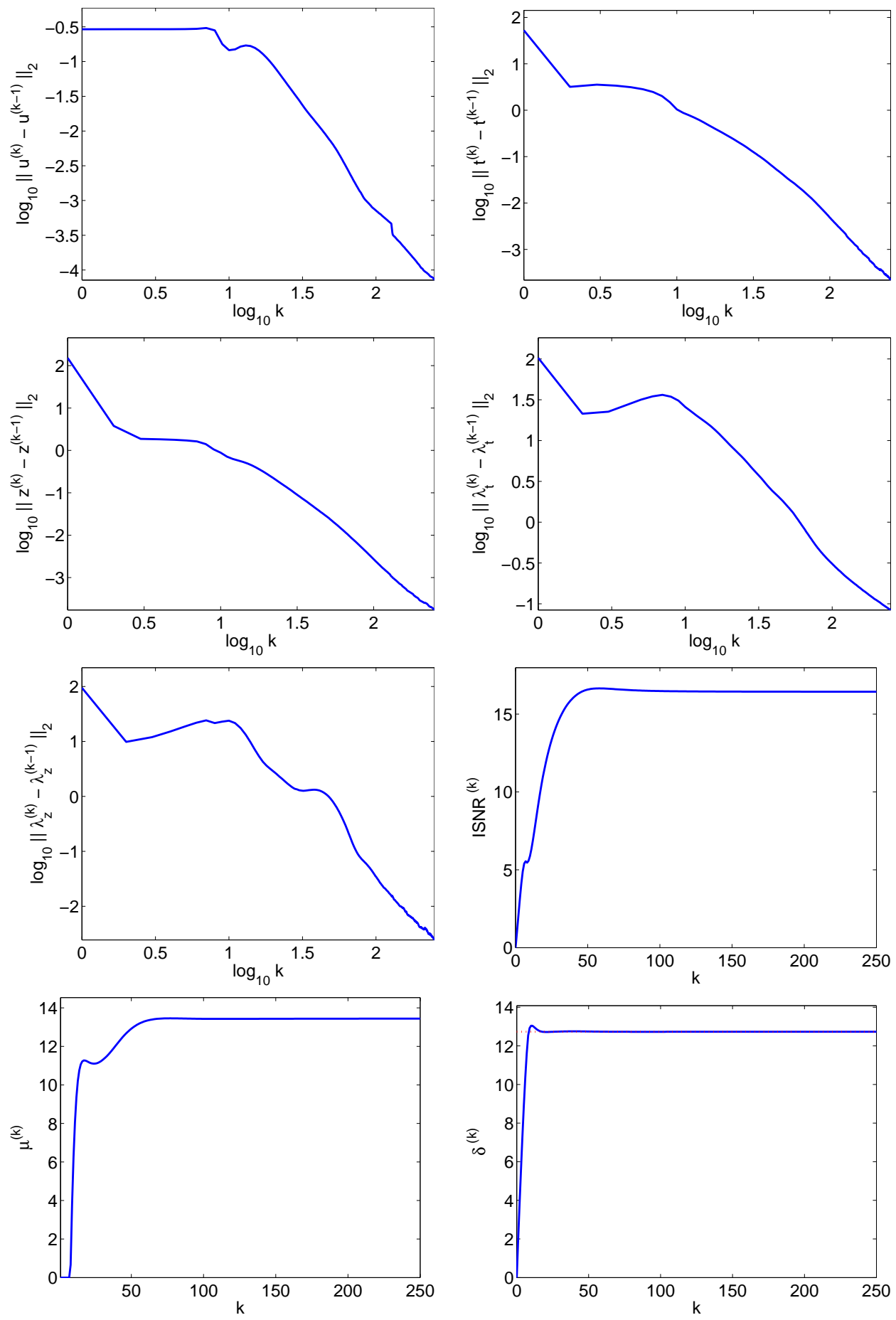

FIG. 5.4. Example 1: plots providing empirical evidence of numerical convergence of the proposed CNC approach. 


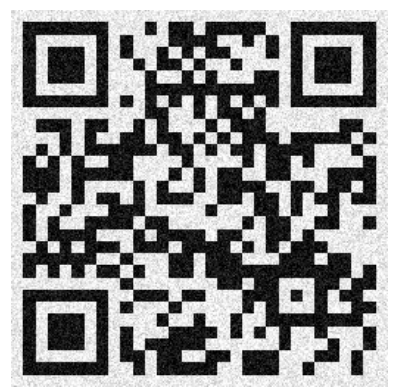

(a) noisy image $\left(\mathrm{SNR}_{b}=15\right)$
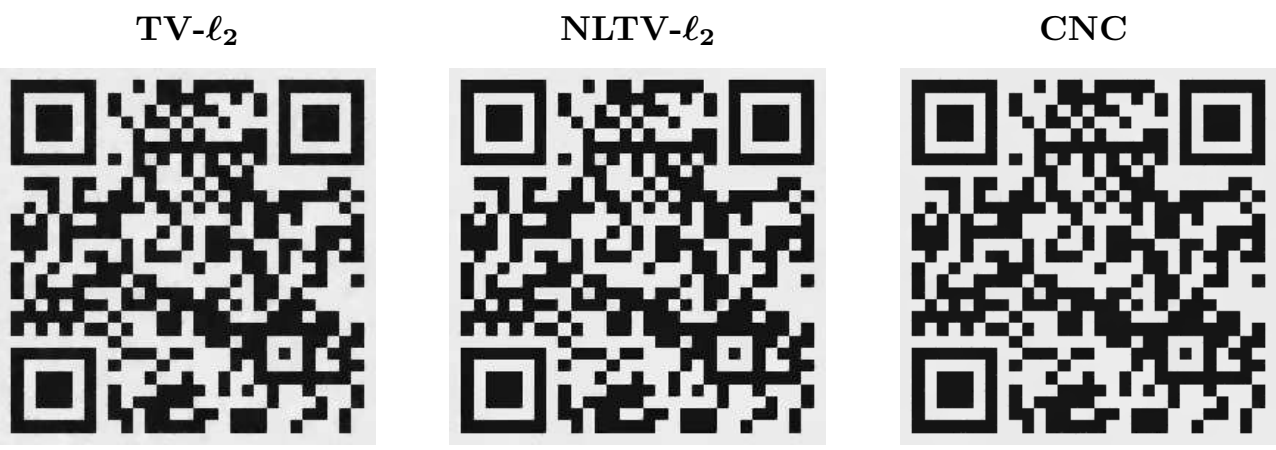

(b) restored images (from left to right, ISNR $=8.61,10.51,15.96$ )
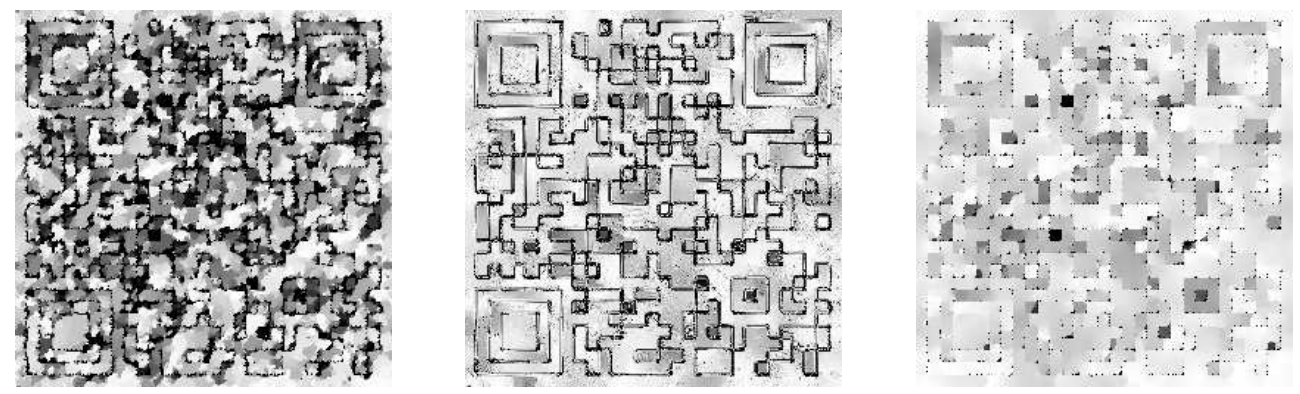

(c) absolute error images

FIG. 5.5. Example 2: Denoising results for test image qrcode corrupted by zero-mean AWGN yielding $S N R_{b}=15$.

to the convexly regularized $\mathrm{TV}-\ell_{2}$ and NLTV- $\ell_{2}$ models are greater for phantom, checkboard and qrcode images. This is not surprising, since these three images are characterized by very sparse gradients (sparser than satellite) and it is wellknown that non-convex regularization holds the potential for inducing sparsity more effectively than convex regularization.

To allow for a visual inspection of the performance, in Figures 5.5 and 5.6 we report some denoising results for the qrcode and phantom images corrupted by AWGN yielding $\mathrm{SNR}_{b}$ equal to 15 and 10, respectively. In particular, in the first row of Figures 5.5 and 5.6 we show the noise-corrupted images whereas in the second row we report the denoised images obtained by the three compared models. In order to better 


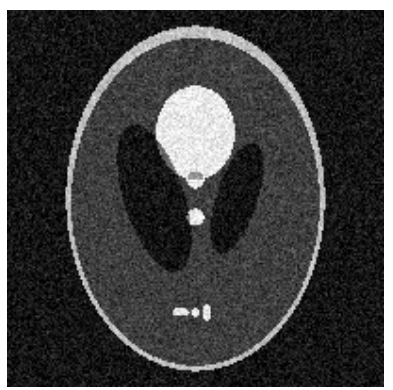

(a) noisy image $\left(\mathrm{SNR}_{b}=10\right)$

$\mathrm{TV}-\ell_{2}$

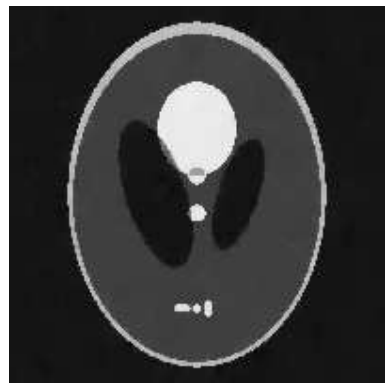

NLTV $-\ell_{2}$

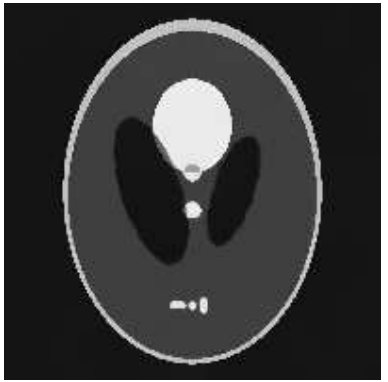

CNC

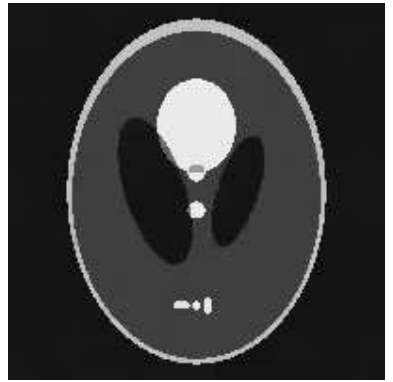

(b) restored images (from left to right, ISNR $=11.86,13.53,17.41$ )
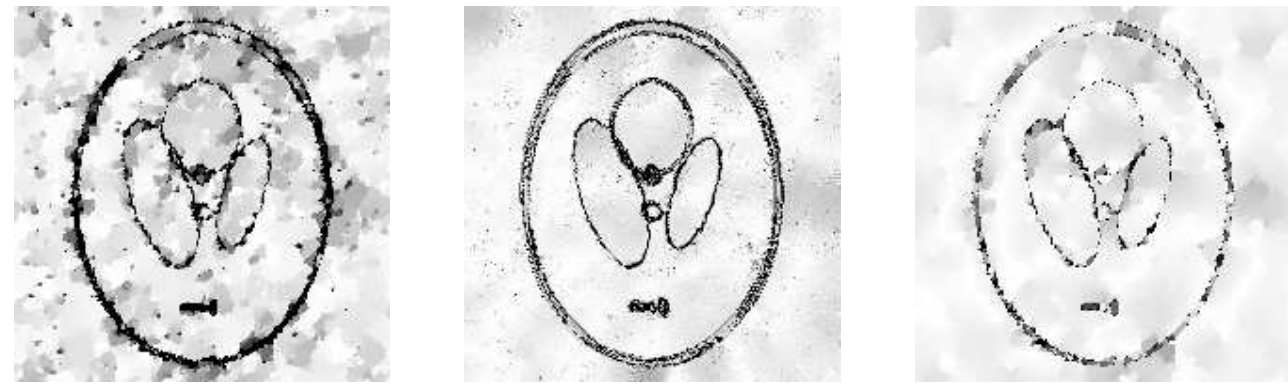

(c) absolute error images

FIG. 5.6. Example 2: Denoising results for test image phantom corrupted by zero-mean AWGN yielding $S N R_{b}=10$.

highlight the differences in the restored images, in the third row of Figures 5.5 and 5.6 we also show the absolute error images given by $\left|\bar{u}-u^{*}\right|$, with $\bar{u}$ and $u^{*}$ denoting the uncorrupted and the restored images, respectively. In particular, the darker are the pixels in the absolute error images, the greater is the error at that pixels in the restored images. It is evident from these error images how the proposed nonconvexly regularized CNC model allows for better restoring edges and details with respect to the convexly-regularized considered models, thus yielding higher ISNR values.

Example 3. In the previous example we have seen how the beneficial effect of using a non-convex versus a convex regularizer is to push the solution closer to the considered uncorrupted test images, which are substantially piecewise constant 
satellite

phantom

\begin{tabular}{|c|c||r|r|r||r|r|r|}
\hline \multicolumn{2}{|c||}{$\mathrm{SNR}_{b}$} & 10 & 15 & 20 & 10 & 15 & 20 \\
\hline \hline \multirow{4}{*}{ ISNR } & TV- $\ell_{2}$ & 8.31 & 7.09 & 6.35 & 11.86 & 11.50 & 11.28 \\
& ${\text { NLTV- } \ell_{2}}$ & 8.45 & 7.55 & 6.88 & 13.53 & 13.49 & 13.32 \\
& $\mathrm{CNC}^{2}$ & $\mathbf{9 . 1 2}$ & $\mathbf{8 . 6 9}$ & $\mathbf{8 . 6 8}$ & $\mathbf{1 7 . 4 1}$ & $\mathbf{1 9 . 6 4}$ & $\mathbf{2 1 . 7 1}$ \\
\hline
\end{tabular}

checkboard

qrcode

\begin{tabular}{|c|c||r|r|r||r|r|r|}
\hline \multicolumn{2}{|c|}{$\mathrm{SNR}_{b}$} & 10 & 15 & 20 & 10 & 15 & 20 \\
\hline \hline \multirow{3}{*}{ ISNR } & TV- $\ell_{2}$ & 7.09 & 6.82 & 6.67 & 8.89 & 8.61 & 8.46 \\
& $\mathrm{NLTV} \ell_{2}$ & 7.37 & 9.53 & 10.33 & 9.42 & 10.51 & 11.18 \\
& $\mathrm{CNC}$ & $\mathbf{1 2 . 2 8}$ & $\mathbf{1 4 . 9 4}$ & $\mathbf{1 6 . 9 4}$ & $\mathbf{1 3 . 9 0}$ & $\mathbf{1 5 . 9 5}$ & $\mathbf{1 7 . 9 9}$ \\
\hline
\end{tabular}

TABLE 5.1

Example 2: ISNR values obtained by denoising the test images satellite and phantom (top), checkboard and qrcode(bottom), corrupted by zero-mean $A W G N$ yielding increasing $S N R_{b}$ values for the $T V-\ell_{2}, N L T V-\ell_{2}$ and $C N C$ approaches.

\begin{tabular}{|c|c||r|r|r|r|r|r|}
\multicolumn{1}{c}{} & \multicolumn{3}{c}{ satellite } & \multicolumn{3}{c|}{ phantom } \\
\hline \multicolumn{2}{|c||}{$\mathrm{SNR}_{b}$} & 10 & 15 & 20 & 10 & 15 & 20 \\
\hline \hline \multirow{3}{*}{ ISNR } & FNNMM & 8.38 & 7.35 & 6.71 & 12.76 & 12.87 & 12.84 \\
& CNC $\left(\tau_{c}=1.1\right)$ & $\mathbf{9 . 1 3}$ & $\mathbf{8 . 7 1}$ & $\mathbf{8 . 7 2}$ & 17.74 & 19.93 & 22.04 \\
& CNC $\left(\tau_{c}=5.0\right)$ & 8.59 & 8.41 & 8.63 & $\mathbf{1 9 . 4 7}$ & $\mathbf{2 2 . 1 7}$ & $\mathbf{2 4 . 3 7}$ \\
\hline
\end{tabular}

checkboard qrcode

\begin{tabular}{|c|c||r|r|r||r|r|r|}
\hline \multicolumn{2}{|c|}{ SNR $_{b}$} & 10 & 15 & 20 & 10 & 15 & 20 \\
\hline \hline \multirow{3}{*}{ ISNR } & FNNMM & 8.59 & 9.23 & 9.79 & 10.09 & 10.70 & 11.15 \\
& CNC $\left(\tau_{c}=1.1\right)$ & 12.59 & 15.34 & 17.15 & 14.24 & 16.26 & 18.11 \\
& CNC $\left(\tau_{c}=5.0\right)$ & $\mathbf{1 6 . 3 1}$ & $\mathbf{1 7 . 6 1}$ & $\mathbf{1 7 . 7 5}$ & $\mathbf{1 7 . 3 3}$ & $\mathbf{1 8 . 7 3}$ & $\mathbf{1 8 . 8 7}$ \\
\hline
\end{tabular}

TABLE 5.2

Example 3: ISNR values obtained by denoising the images satellite and phantom (top), checkboard and qrcode(bottom), corrupted by zero-mean $A W G N$ yielding increasing $S N R_{b}$ values for the FNNMM and CNC approaches.

images. These effect is quite well known and comes from the ability of non-convex regularizers to induce with more strength the sparsity of gradient magnitudes in the restored image, as theoretically analyzed in [19]. Moreover, the results in Example 1 indicate that for images characterized by a degree of sparsity (of gradient magnitudes) similar to the considered test images, more accurate restorations are obtained by pushing the functional towards its convexity limit, i.e. by letting the convexity coefficient $\tau_{c}$ tend to 1 from the left.

In this example, we investigate the potential of our model when $\tau_{c}>1$ which, according to results in Section 3, becomes non-convex. In particular, to better evaluate the performance of our model we compare it with the state-of-the-art non-convex 
non-smooth variational model proposed in [22], minimized by a non-smooth Graduated Non-convexity Continuation (GNC) approach. The model will be referred to as FNNMM. In particular, the non-convex rational prior used in [22] depends on a parameter $\alpha$ analogous to our parameter $a$. Since the authors did not provide any procedure to automatically estimate $\alpha$, we used the suggested value $\alpha=0.5$ for all the experiments.

In Table 5.2 we report the results of the comparison, where, analogously to Table $5.1, \mathrm{SNR}_{b}$ denotes the SNR of the noise-corrupted image $b$ and the best ISNR results are marked in boldface. From Table 5.2 we can observe that for $\tau_{c}>1$ the restoration results obtained by our model outperform both the results achieved by our model in the convex regime $\left(\tau_{c}=0.99\right)$ reported in Table 5.1 and the ISNR values of FNNMM restorations. More precisely, in Table 5.2 we show the ISNR values achieved by our model for two different values of $\tau_{c}$, namely $\tau_{c} \in\{1.1,5.0\}$. We notice that the ISNR values for $\tau_{c}=5$ turn out to be higher than for $\tau_{c}=1.1$ except for the satellite image, as expected due to its previously outlined characteristics.

The conclusion of the experimental investigation carried out above is that pure non-convex models hold the potential for obtaining higher quality restorations than convex (or CNC) models for images characterized by very sparse gradients, such as the considered test images phantom, checkboard and qrcode. In this case, the proposed model (1.4) with $\tau_{c}>1$ compares successively with other non-convex state-of-the-art approaches. However, as well known, these models need to be used carefully due to numerical and computational issues arising as a consequence of their non-convexity. It is worth recalling that no minimization algorithm guarantees the finding of the global minimum of a general non-convex cost-function and thus the obtained solutions are in general only local minimizers.

In the following, we present a final experimental test aimed at demonstrating that for certain types of images our CNC proposal can be an optimal choice in terms of achieved restoration accuracy with respect not only to pure convex models but also to pure non-convex models. At this aim, we consider the two test images satellite and qrcode and a new test image cameraman, shown in the first row of Figure 5.7. In the second and third row of Figure 5.7 we report the normalized histograms of the gradient magnitudes of the images and zoomed details of such histograms, respectively. In the last row, we show plots representing the restoration quality (ISNR) achieved by the proposed model (1.4) applied to the three considered images for different values of the convexity coefficient $\tau_{c}$, both in the pure convex $\left(\tau_{c}=0\right.$, i.e. ROF model) or CNC $\left(0<\tau_{c} \leq 1\right)$ and in the pure non-convex $\left(\tau_{c}>1\right)$ regimes. It can be observed that for the qrcode image, as previously pointed out, the best restorations are obtained by pushing the model into the pure non-convex regime. In fact, qrcode is a representative for the class of piecewise constant image characterized by very sparse gradients histograms. The satellite test image can be considered as less piecewise constant than qrcode, with a much more spread out histogram. In this case, the most accurate restorations are obtained near the convexity limit $\tau_{c}=1$, as visible in the ISNR plot in the last row of Figure 5.7. Finally, cameraman can be representative for photographic images which present a wide gradients histogram with significative homogeneous regions which benefit of the non-convex regularization term in the model. For this image, the best restorations are achieved for $\tau_{c}$ in the range $[0.3,0.5]$, that is by using the proposed model (1.4) in the CNC regime.

Our CNC model has adavantages for the class of images which are no longer purely piecewise constant, but present several homogeneous regions, for which the 
restoration would be penalized by both using a pure non-convex model and also by using a pure convex model. This would suggest a future direction of investigation to adapt the convexity of the model to the content of the images.
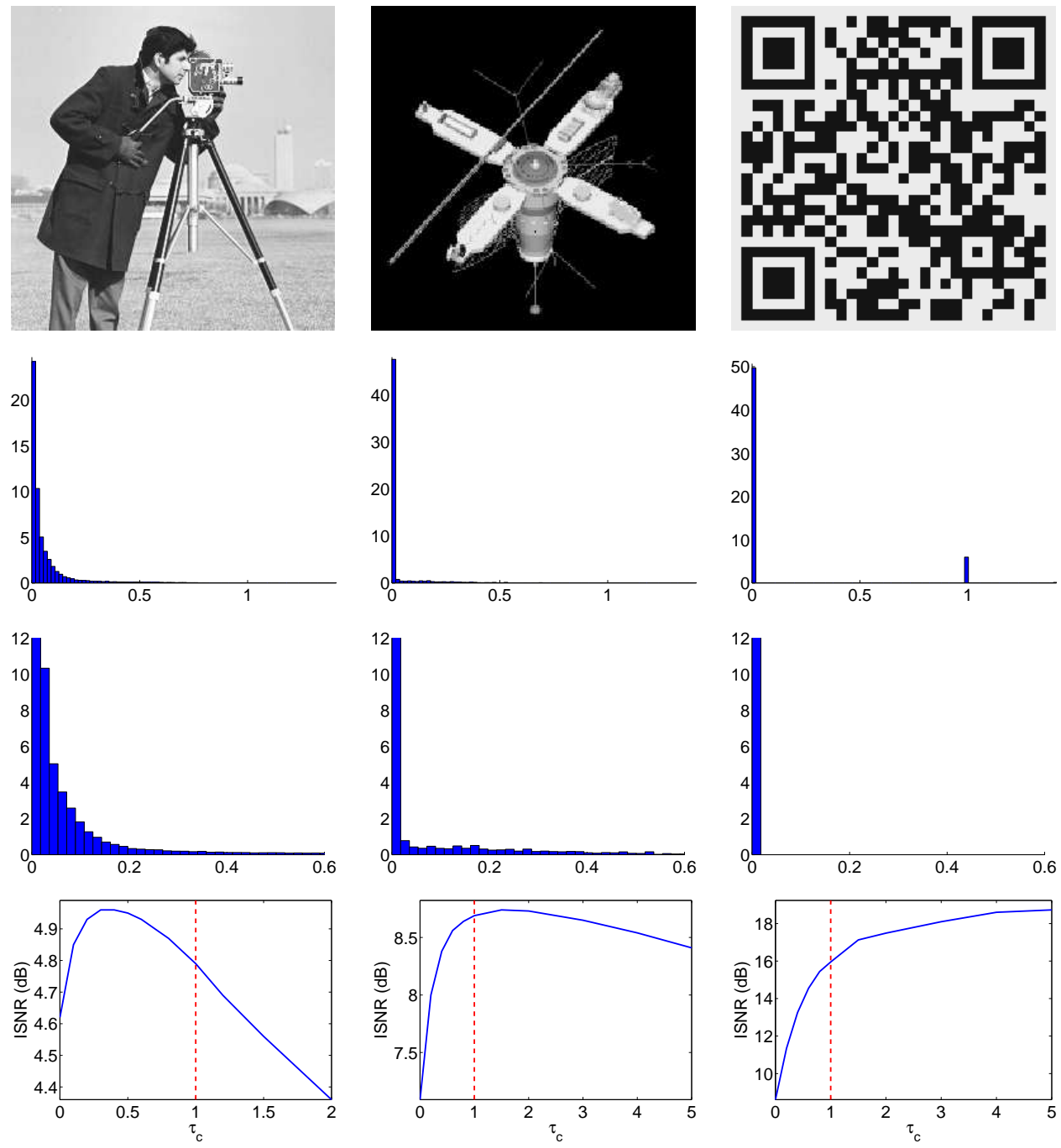

FIG. 5.7. Example 3. First row: the three images cameraman (left), satellite (center) and qrcode (right). Second row: the associated normalized histograms of gradient magnitudes. Third row: zoomed details of the histograms. Last row: restoration quality (in terms of ISNR values) achieved by the proposed variational model (1.4) applied to the three considered images for different values of the convexity coefficient $\tau_{c}$, both in the convex $\left(0 \leq \tau_{c} \leq 1\right)$ and in the non-convex $\left(\tau_{c}>1\right)$ regime.

6. Conclusions. We presented a CNC variational model for image denoising which includes a parameterized non-convex non-smooth regularization term aimed at forcing sparsity of gradients in the restored image. An automatic strategy for the selection of the regularization parameter based on the discrepancy principle has been integrated into an efficient ADMM-based minimization algorithm. Theoretical convexity conditions for the considered objective functional as well as for the optimization 
sub-problems arising in the ADMM-based iterative scheme have been derived, which guarantee the existence of a unique global minimizer. A novel non-convex exponential penalty function has been proposed which allows for improved restorations and for a closed-form threshold function. Numerical results on sparse-gradient images strongly indicate that the proposed CNC model outperforms classical convex models with non-smooth but convex regularizers, such as TV- $\ell_{2}$ and NLTV- $\ell_{2}$. The proposed model has been tested also in the non-convex regime and successfully compared with a state-of-the-art non-convex method. In conclusion, the introduced model can be flexibly deployed with high quality results both in the convex and non-convex setting. Moreover, the proposed approach is not limited to the image denoising application, but it is general enough to be used in other important fields. This will be matter of future work.

\section{REFERENCES}

[1] J. Bioucas-Dias, and M. Figueredo, Fast image Recovery Using Variable Splitting and Constrained Optimization, IEEE Transaction on Image Processing, 19(9), pp. 2345-2356, 2010.

[2] A. Blake, A. Zisserman, Visual Reconstruction, Cambridge, MA, USA: MIT Press, 1987.

[3] S. Boyd, N. Parikh, E. Chu, B. Peleato, and J. Eckstein, Distributed Optimization and Statistical Learning via the Alternating Direction Method of Multipliers, Foundations and Trends in Machine Learning, vol. 3(1), pp. 1122, 2011.

[4] D. Calvetti and L. Reichel, Tikhonov regularization of large linear problems, BIT Numer. Math., 43 (2003), pp. 263-283.

[5] D. Calvetti, S. Morigi, L. Reichel, and F. Sgallari, Tikhonov regularization and the L-curve for large, discrete ill-posed problems, J. Comput. Appl. Math., 123 (2000), pp. 423-446.

[6] R.H. Chan, M. Tao and X.M. Yuan, Constrained Total Variational Deblurring Models and Fast Algorithms Based on Alternating Direction Method of Multipliers, SIAM J. Imag. Sci., 6, pp. 680-697, (2013)

[7] P.Y. Chen, I.W. Selesnick, Group-Sparse Signal Denoising: Non-Convex Regularization, Convex Optimization, IEEE Trans. on Sign. Proc., 62 (2014) pp. 3464-3478.

[8] M. Christiansen and M. Hanke, Deblurring Methods Using Antireflective Boundary Conditions, SIAM Journal on Scientific Computing, 30, pp. 855-872, 2008.

[9] F. H. Clarke, Optimizatiom and Nonsmooth Analysis, Wiley, 1983.

[10] R.M. Corless, G.H. Gonnet, D.E.G. Hare, D.J. Jeffrey, and D.E. Knuth, On the Lambert W Function, Advances in Computational Mathematics, vol.5, pp. 329-359, 1996.

[11] I. Ekeland and R. Temam, Convex Analysis and Variational Problems (Classics in Applied Mathematics). Philadelphia, PA, USA: SIAM, 1999.

[12] H. W. Engl, M. Hanke, and A. Neubauer, Regularization of Inverse Problems, Kluwer, Dordrecht, The Netherlands, 1996.

[13] R. Glowinski, and P. Le Tallec, Augmented Lagrangians and Operator-Splitting Methods in Nonlinear Mechanics, SIAM, Philadelphia, 1989.

[14] C. He, C. Hu, W. Zhang, B. Shi, A Fast Adaptive Parameter Estimation for Total Variation Image Restoration, Image Processing, IEEE Transactions on , 23(21), pp. 4954-4967, 2014.

[15] A. Lanza, S. Morigi, F. Sgallari, Convex Image Denoising via Non-Convex Regularization, Scale Space and Variational Methods in Computer Vision, 9087, pp. 666-677, 2015.

[16] C. W. Lu, Image restoration and decomposition using nonconvex nonsmooth regularisation and negative Hilbert-Sobolev norm, IET Image Processing, 6(6), pp. 706-716, (2012)

[17] M. K. Ng, R. H. Chan, and W. C. Tang, A Fast Algorithm for Deblurring Models with Neumann Boundary Conditions, SIAM Journal on Scientific Computing, 21, pp. 851-866, 1999.

[18] M. Nikolova, Estimation of Binary Images by Minimizing Convex Criteria, in Proc. IEEE Int. Conf. Image Process., 2, pp. 108-112, 1998.

[19] M. Nikolova, Analysis of the Recovery of Edges in Images and Signals by Minimizing Nonconvex Regularized Least-Squares, Multiscale Modeling and Simulation, pp. 960-991, 4(3), 2005.

[20] M. Nikolova, M. Ng, and C. Tam, Software is available at http://www. math.hkbu.edu.hk/ mng/imaging-software.html

[21] M. Nikolova, Ng, M., Tam,C.P., On $\ell_{1}$ Data Fitting and Concave Regularization for Image Recovery, SIAM J. on Scientific Computing, 35/1, pp. A397-A430 (2013)

[22] M. Nikolova, M. K. Ng and C. P. Tam, Fast Non-convex Non-smooth Minimization Methods 
for Image Restoration and Reconstruction, IEEE Trans. Image Processing, 19(12), pp. 30733088, (2010)

[23] A. Parekh, I.W. Selesnick, Convex Denoising using Non-Convex Tight Frame Regularization arXiv preprint arXiv:1504.00976, 2015

[24] R. T. Rockafellar, Convex Analysis, Princeton Univ. Press, Princeton, N.J., 1970.

[25] R. T. Rockafellar and R. J. B. Wets, Variational Analysis, vol. 317 of Grundlehren der Mathematischen Wissenschaften [Fundamental Principles of Mathematical Sciences], Springer, Berlin, Germany, 1998.

[26] L.I. Rudin, S. Osher and E. Fatemi, Nonlinear total variation based noise removal algorithms, Physics D, 60(1-4), pp. 259-268, 1992.

[27] I. W. Selesnick, I. Bayram, Sparse Signal Estimation by Maximally Sparse Convex Optimization, IEEE Trans. on Signal Proc., 62(5) (2014) pp. 1078-1092.

[28] I. W. Selesnick, A. Parekh, and I. Bayram, Convex 1-D total variation denoising with non-convex regularization, IEEE Signal Proc. Letters, 22(2) (2015) pp. 141-144.

[29] E. Y. Sidky, R. Chartrand, J. M. Boone, X. Pan, Constrained TpV-Minimization for Enhanced Exploitation of Gradient Sparsity: Application to CT Image Reconstruction, IEEE Journal of Translational Engineering in Health and Medicine, 2, pp. 1-18, 2014.

[30] Y.W. Wen, R.H. Chan, Parameter selection for total-variation-based image restoration using discrepancy principle, IEEE Transactions on Image Processing, 21(4), pp. 1770-1781, 2012.

[31] C. Wu and X.C. Tai, Augmented Lagrangian Method, Dual Methods, and Split Bregman Iteration for ROF, Vectorial TV, and High Order Models, SIAM Journal on Imaging Sciences, 3(3), pp. 300-339, 2010.

[32] X. Zhang, M. Burger, X. Bresson, and S. Osher, Bregmanized Nonlocal Regularization for Deconvolution and Sparse Reconstruction, SIAM Journal on Imaging Sciences, 3(3), pp. 253-276, 2010

[33] C. Wu, J. Zhang and X.C. Tai, Augmented Lagrangian Method for Total Variation Restoration with Non-Quadratic Fidelity, Inverse Problems and Imaging, 5(1), pp. 237-261, 2011.

\section{APPENDIX. Proof of Proposition 3.2.}

Proof. Recalling that convexity of a function is invariant to non singular linear transformations of the domain, we search for a non singular linear transformation $T: \mathbb{R}^{3} \rightarrow \mathbb{R}^{3}$ of the domain of function $f$ defined in (3.7), that is $x=T y$, with $x=\left(x_{1}, x_{2}, x_{3}\right)^{T}, y=\left(y_{1}, y_{2}, y_{3}\right)^{T}, T=\left(T_{i, j}\right)_{i, j:=1,2,3}$ such that convexity conditions for the function $f_{T}:=f \circ T$ are easier to identify than for $f$. We obtain the explicit expression of function $f_{T}$, depending on $y$ and on the nine entries of the transformation matrix $T$, by replacing $x=T y$ in (3.7):

$$
f_{T}(y ; T):=f(T y)=\mu Q_{1}(y ; T)+\phi\left(\frac{1}{\Delta} \sqrt{Q_{2}(y ; T)} ; a\right),
$$

where $Q_{1}$ and $Q_{2}$ are quadratic functions. We impose that both $Q_{1}$ and $Q_{2}$ do not contain mixed products, that $Q_{2}$ does not depend on $y_{3}$ and that the coefficients of both $y_{1}^{2}$ and $y_{2}^{2}$ in $Q_{2}$ are equal to one; we obtain that the transformation matrix $T=(0, \sqrt{2} / 3, \sqrt{3} / 3 ; \sqrt{2} / 2,-\sqrt{2} / 6, \sqrt{3} / 3 ;-\sqrt{2} / 2,-\sqrt{2} / 6, \sqrt{3} / 3)$ yields:

$$
f_{T}\left(y_{1}, y_{2}, y_{3}\right)=\frac{\mu}{6}\left(y_{1}^{2}+y_{2}^{2}+y_{3}^{2}\right)+\phi\left(\frac{1}{\Delta} \sqrt{y_{1}^{2}+y_{2}^{2}}\right) .
$$

It follows that the function $f_{T}\left(y_{1}, y_{2}, y_{3}\right)$ above, hence also $f$ in (3.7), are strictly convex if and only if the function $g\left(y_{1}, y_{2}\right)$ defined in (3.9) is strictly convex. 


\section{Proof of Proposition 3.3.}

Proof. The function $g: \mathbb{R}^{2} \rightarrow \mathbb{R}$ in (3.9) can be rewritten in composite form as follows:

$$
g\left(y_{1}, y_{2}\right)=h\left(\rho\left(y_{1}, y_{2}\right)\right)
$$

with the function $\rho: \mathbb{R}^{2} \rightarrow \mathbb{R}_{+}$defined by

$$
\rho\left(y_{1}, y_{2}\right)=\sqrt{y_{1}^{2}+y_{2}^{2}},
$$

and the function $h: \mathbb{R}_{+} \rightarrow \mathbb{R}$ defined in (3.10).

We notice that, due to definition of function $\rho$ in (6.4) and due to Assumption 1) in Section 2 on the penalty function $\phi$, we have:

$$
\rho \in \mathcal{C}^{0}\left(\mathbb{R}^{2}\right) \cap \mathcal{C}^{2}\left(\mathbb{R}^{2} \backslash\{(0,0)\}\right), \quad h \in \mathcal{C}^{0}\left(\mathbb{R}_{+}\right) \cap \mathcal{C}^{2}\left(\mathbb{R}_{+}^{*}\right) .
$$

It follows from (6.3)-(6.5) that the function $g$ is such that

$$
g \in \mathcal{C}^{0}\left(\mathbb{R}^{2}\right) \cap \mathcal{C}^{2}\left(\mathbb{R}^{2} \backslash\{(0,0)\}\right) .
$$

Hence, condition for $g$ to be strictly convex is that its Hessian matrix $H_{g}\left(y_{1}, y_{2}\right)$ is positive definite for any $\left(y_{1}, y_{2}\right) \in \mathbb{R}^{2} \backslash\{(0,0)\}$. In the following, we investigate such condition.

By applying the chain rule of differentiation twice to the function $g$ in composite form (6.3), we get:

$$
H_{g}=H_{\rho} h^{\prime}+\nabla \rho \nabla \rho^{T} h^{\prime \prime}=\left(\begin{array}{cc}
\rho_{1,1} h^{\prime}+\rho_{1}^{2} h^{\prime \prime} & \rho_{1,2} h^{\prime}+\rho_{1} \rho_{2} h^{\prime \prime} \\
\rho_{1,2} h^{\prime}+\rho_{1} \rho_{2} h^{\prime \prime} & \rho_{2,2} h^{\prime}+\rho_{2}^{2} h^{\prime \prime}
\end{array}\right),
$$

where $H_{\rho}$ and $\nabla \rho$ denote the Hessian matrix and the gradient of function $\rho$ in (6.4), respectively, and where, for simplicity of notations, dependencies on independent variables are dropped and a concise notation for ordinary and partial derivatives is adopted, namely $h^{\prime}:=d h / d \rho, h^{\prime \prime}:=d^{2} h / d \rho^{2}, \rho_{i}:=\partial \rho / \partial y_{i}, \rho_{i, j}:=\partial^{2} \rho / \partial y_{i} \partial y_{j}$, $i, j \in\{1,2\}$. We remark that, since we are considering the case $\left(y_{1}, y_{2}\right) \neq(0,0)$, all the differential quantities in (6.7) are well defined and, in particular, no one-sided derivative is involved.

According to the Sylvester's criterion, the Hessian matrix $H_{g}$ in (6.7) is positive definite if and only if its two leading principal minors are positive, that is if the following two conditions hold:

$$
\left\{\begin{array}{l}
\rho_{1,1} h^{\prime}+\rho_{1}^{2} h^{\prime \prime}>0 \\
\left(\rho_{1,1} \rho_{2,2}-\rho_{1,2}^{2}\right)\left(h^{\prime}\right)^{2}+\left(\rho_{2,2} \rho_{1}^{2}+\rho_{1,1} \rho_{2}^{2}-2 \rho_{1} \rho_{2} \rho_{1,2}\right) h^{\prime} h^{\prime \prime}>0
\end{array} .\right.
$$

The first and second order partial derivatives of $\rho$ in (6.4) are as follows:

$$
\rho_{1}=\frac{y_{1}}{\rho}, \quad \rho_{2}=\frac{y_{2}}{\rho}, \quad \rho_{1,1}=\frac{y_{2}^{2}}{\rho^{3}}, \quad \rho_{2,2}=\frac{y_{1}^{2}}{\rho^{3}}, \quad \rho_{1,2}=-\frac{y_{1} y_{2}}{\rho^{3}} .
$$


Replacing expressions (6.9) into $H_{g}$ positive definiteness conditions (6.8) and recalling that $\rho$ is a positive quantity for every $\left(y_{1}, y_{2}\right) \neq(0,0)$, we obtain:

$$
\begin{aligned}
& \left\{\begin{array}{l}
\frac{y_{2}^{2}}{\rho^{3}} h^{\prime}+\frac{y_{1}^{2}}{\rho^{2}} h^{\prime \prime}>0 \\
\left(\frac{y_{1}^{2} y_{2}^{2}}{\rho^{6}}-\frac{y_{1}^{2} y_{2}^{2}}{\rho^{6}}\right)\left(h^{\prime}\right)^{2}+\left(\frac{y_{1}^{4}}{\rho^{5}}+\frac{y_{2}^{4}}{\rho^{5}}+2 \frac{y_{1}^{2} y_{2}^{2}}{\rho^{5}}\right) h^{\prime} h^{\prime \prime}>0
\end{array} \equiv\right. \\
& \equiv\left\{\begin{array} { l } 
{ y _ { 2 } ^ { 2 } h ^ { \prime } + \rho y _ { 1 } ^ { 2 } h ^ { \prime \prime } > 0 } \\
{ \frac { ( y _ { 1 } ^ { 2 } + y _ { 2 } ^ { 2 } ) ^ { 2 } } { \rho ^ { 5 } } h ^ { \prime } h ^ { \prime \prime } > 0 }
\end{array} \equiv \left\{\begin{array} { l } 
{ y _ { 2 } ^ { 2 } h ^ { \prime } + \rho y _ { 1 } ^ { 2 } h ^ { \prime \prime } > 0 } \\
{ h ^ { \prime } h ^ { \prime \prime } > 0 }
\end{array} \equiv \left\{\begin{array}{l}
h^{\prime}>0 \\
h^{\prime \prime}>0
\end{array}\right.\right.\right. \text {. }
\end{aligned}
$$

Hence, the function $g$ defined in (3.9) is strictly convex if and only if the function $h$ in (3.10) is monotonically increasing and strictly convex.

\section{Proof of Proposition 4.2.}

Proof. According to Proposition (3.5), in case that the parameters pair $(\mu, a)$ satisfies (3.13), the functional $\mathcal{J}(u ; \mu, a)$ in (1.4) is strictly convex, thus admitting a unique minimizer $u^{*}$. Then, the first-order optimality condition for $\mathcal{J}$ at $u^{*}$ given in (4.6) follows immediately from the generalized Fermat's rule (see Theorem 10.1 in $[25])$.

To prove (4.7), we need to write in a more explicit form the subdifferential $\partial_{u}[\mathcal{J}]$. However, we can not apply the additive rule of subdifferentials to the functional $\mathcal{J}$ since the regularization term $\Phi$ in (4.5) is non-convex in $u$ due to concavity of the penalty function $\phi$. Hence, we resort to notions from calculus for non-smooth nonconvex functions, in particular the Clarke generalized gradient [9], which extends the concept of subdifferential for non-smooth convex functions to the case of non-smooth non-convex but locally Lipschitz functions. Indeed, the rest of the proof relies on the fact that, according to Lemma 4.1, both the total functional $\mathcal{J}$ in (1.4) and, separately, the $\Phi$ regularization term in (4.5) and the quadratic fidelity term, are locally Lipschitz functions, such that their generalized gradient is defined.

First, we recall that for non-smooth but convex functions the Clarke generalized gradient is equal to the subdifferential [9], that is, in our case:

$$
\partial_{u}[\mathcal{J}]\left(u^{*}\right)=\bar{\partial}_{u}[\mathcal{J}]\left(u^{*}\right) .
$$

After recalling the definition of functional $\mathcal{J}$ in (1.4), we can now apply the additive rule of generalized gradients [9] to the right-hand side of (6.11):

$$
\begin{aligned}
\bar{\partial}_{u}[\mathcal{J}]\left(u^{*}\right) & \subset \bar{\partial}_{u}[\Phi]\left(u^{*}\right)+\bar{\partial}_{u}\left[\frac{\mu}{2}\|u-b\|_{2}^{2}\right]\left(u^{*}\right) \\
& =\bar{\partial}_{u}[\Phi]\left(u^{*}\right)+\mu\left(u^{*}-b\right),
\end{aligned}
$$

where in (6.12) we applied the property that the generalized gradient reduces to the usual gradient in case of continuously differentiable functions.

Recalling the definition of the $\Phi$ regularization term in (4.5) and applying to the first term of (6.12) the chain rule for generalized gradients [9], we obtain:

$$
\bar{\partial}_{u}[\Phi]\left(u^{*}\right)=D^{T} \bar{\partial}_{D u}[\Phi]\left(D u^{*}\right) .
$$


From (6.13), (6.12), (6.11) and statement (4.6), statement (4.7) follows immediately.

口

\section{Proof of Theorem 4.4.}

Proof. Based on the definition of the augmented Lagrangian functional in (4.3), we rewrite in explicit form the first inequality of the saddle-point condition in (4.4):

$$
\begin{aligned}
& \sum_{i=1}^{n} \phi\left(H t_{i}^{*} \|_{2} ; a\right)+\frac{\mu}{2}\left\|z^{*}-\delta\right\|_{2}^{2}+\frac{\beta_{z}}{2}\left\|z^{*}-u^{*}\right\|_{2}^{2}+\frac{\beta_{t}}{2}\left\|t^{*}-D u^{*}\right\|_{2}^{2} \\
& -\left\langle\lambda z, z^{*}-u^{*}\right\rangle-\left\langle\lambda_{t}, t^{*}-D u^{*}\right\rangle \\
\leq & \sum_{i=1}^{n} \phi\left(H t_{i}^{*} \|_{2} ; a\right)+\frac{\mu}{2}\left\|z^{*}-\delta\right\|_{2}^{2}+\frac{\beta_{z}}{2}\left\|z^{*}-u^{*}\right\|_{2}^{2}+\frac{\beta_{t}}{2}\left\|t^{*}-D u^{*}\right\|_{2}^{2} \\
& -\left\langle\lambda_{z}^{*}, z^{*}-u^{*}\right\rangle-\left\langle\lambda_{t}^{*}, t^{*}-D u^{*}\right\rangle \quad \forall\left(\lambda_{z}, \lambda_{t}\right) \in V \times Q \\
\equiv & \left\langle\lambda_{z}^{*}-\lambda_{z}, z^{*}-u^{*}\right\rangle+\left\langle\lambda_{t}^{*}-\lambda_{t}, t^{*}-D u^{*}\right\rangle \leq 0 \quad \forall\left(\lambda_{z}, \lambda_{t}\right) \in V \times Q,(
\end{aligned}
$$

and, similarly, the second inequality:

$$
\begin{aligned}
& \sum_{i=1}^{n} \phi\left(\left\|t_{i}^{*}\right\|_{2} ; a\right)+\frac{\mu}{2}\left\|z^{*}-b\right\|_{2}^{2}+\frac{\beta_{z}}{2}\left\|z^{*}-u^{*}\right\|_{2}^{2}+\frac{\beta_{t}}{2}\left\|t^{*}-D u^{*}\right\|_{2}^{2} \\
& -\left\langle\lambda_{z}^{*}, z^{*}-u^{*}\right\rangle-\left\langle\lambda_{t}^{*}, t^{*}-D u^{*}\right\rangle \\
& \leq \sum_{i=1}^{n} \phi\left(\left\|t_{i}\right\|_{2} ; a\right)+\frac{\mu}{2}\|z-b\|_{2}^{2}+\frac{\beta_{z}}{2}\|z-u\|_{2}^{2}+\frac{\beta_{t}}{2}\|t-D u\|_{2}^{2}
\end{aligned}
$$

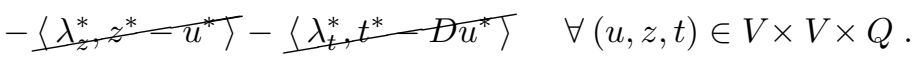

In the first part of the proof, we demonstrate that if $\left(u^{*}, z^{*}, t^{*} ; \lambda_{z}^{*}, \lambda_{t}^{*}\right)$ is a solution of the saddle-point problem (4.3)-(4.4), that is it satisfies the two inequalities (6.14) and (6.15), then $u^{*}$ is the unique minimizer of the functional $\mathcal{J}(u ; \mu, a)$ in (1.4).

Since (6.14) must be satisfied for any $\left(\lambda_{z}, \lambda_{t}\right) \in V \times Q$, by taking $\lambda_{z}=\lambda_{z}^{*}$ we obtain:

$$
\left\langle\lambda_{t}^{*}-\lambda_{t}, t^{*}-D u^{*}\right\rangle \leq 0 \quad \forall \lambda_{t} \in Q \quad \Longrightarrow \quad t^{*}=D u^{*} .
$$

Similarly, by taking $\lambda_{t}=\lambda_{t}^{*}$ in (6.14) we have:

$$
\left\langle\lambda_{z}^{*}-\lambda_{z}, z^{*}-u^{*}\right\rangle \leq 0 \quad \forall \lambda_{z} \in V \quad \Longrightarrow \quad z^{*}=u^{*} .
$$

The second inequality (6.15) must be satisfied for any $(u, z, t) \in V \times V \times Q$. Hence, by taking simultaneously $z=u$ and $t=D u$ in (6.15) and, at the same time, substituting in (6.15) the two previously derived conditions (6.16) and (6.17), we obtain:

$$
\begin{aligned}
& \sum_{i=1}^{n} \phi\left(\left\|\left(D u^{*}\right)_{i}\right\|_{2} ; a\right)+\frac{\mu}{2}\left\|u^{*}-b\right\|_{2}^{2} \\
\leq & \sum_{i=1}^{n} \phi\left(\left\|(D u)_{i}\right\|_{2} ; a\right)+\frac{\mu}{2}\|u-b\|_{2}^{2} \quad \forall u \in V .
\end{aligned}
$$

Inequality (6.18) indicates that $u^{*}$ is a global minimizer of the functional $\mathcal{J}(u ; \mu, a)$ in (1.4). Hence, we have demonstrated that all the saddle-point solutions, if there exists 
one, of problem (4.3)-(4.4) are of the form $\left(u^{*}, u^{*}, D u^{*} ; \lambda_{z}^{*}, \lambda_{t}^{*}\right)$, with $u^{*}$ denoting the unique global minimizer of $\mathcal{J}(u ; \mu, a)$.

In the second part of the proof, we demonstrate that at least one solution of the saddle-point problem exists. In particular, we prove that if $u^{*}$ is a minimizer of $\mathcal{J}(u ; \mu, a)$ in $(1.4)$, then there exist $\left(z^{*}, t^{*}\right) \in V \times Q$ and $\left(\lambda_{z}^{*}, \lambda_{t}^{*}\right) \in V \times Q$ such that $\left(u^{*}, z^{*}, t^{*} ; \lambda_{z}^{*}, \lambda_{t}^{*}\right)$ is a solution of the saddle-point problem (4.3)-(4.4), that is it satisfies the two inequalities (6.14) and (6.15). The demonstration relies on an initial suitable choice of the vectors $z^{*}, t^{*}, \lambda_{z}^{*}$ and $\lambda_{t}^{*}$. Analogously to the proofs in [31], [14], we take:

$$
\begin{aligned}
z^{*}=u^{*}, & t^{*}=D u^{*}, \\
\lambda_{z}^{*}=\mu\left(u^{*}-b\right), & \lambda_{t}^{*} \in \bar{\partial}_{D u}[\Phi]\left(D u^{*}\right) \text { s.t. }: \quad \lambda_{z}^{*}+D^{T} \lambda_{t}^{*}=0,
\end{aligned}
$$

where the term $\bar{\partial}_{D u}[\Phi]\left(D u^{*}\right)$ indicates the Clarke generalized gradient (with respect to $D u$, calculated at $\left.D u^{*}\right)$ of the non-convex regularization term $\Phi$ in (4.5). We notice that a vector $\lambda_{t}^{*}$ satisfying (6.20) is guaranteed to exist thanks to Proposition 4.2. In fact, since here we are assuming that $u^{*}$ is a minimizer of functional $\mathcal{J}(u ; \mu, a)$, the first-order optimality condition in (4.7) holds true.

Due to the first two settings in (6.19), the first saddle-point condition in (6.14) is clearly satisfied. Proving the second condition, that we rewrite in compact form as

$$
\mathcal{L}\left(u^{*}, z^{*}, t^{*} ; \lambda_{z}^{*}, \lambda_{t}^{*} ; \mu, a\right) \leq \mathcal{L}\left(u, z, t ; \lambda_{z}^{*}, \lambda_{t}^{*} ; \mu, a\right) \quad \forall(u, z, t) \in V \times V \times Q,
$$

is less straightforward: we need to investigate the optimality conditions of functional $\mathcal{L}\left(u, z, t ; \lambda_{z}^{*}, \lambda_{t}^{*} ; \mu, a\right)$ in (6.21). To this aim, we introduce below the three functions $\mathcal{L}_{u}\left(u, z, t ; \lambda_{z}^{*}, \lambda_{t}^{*} ; \mu, a\right), \mathcal{L}_{z}\left(u, z, t ; \lambda_{z}^{*}, \lambda_{t}^{*} ; \mu, a\right)$ and $\mathcal{L}_{t}\left(u, z, t ; \lambda_{z}^{*}, \lambda_{t}^{*} ; \mu, a\right)$ representing the restriction of $\mathcal{L}\left(u, z, t ; \lambda_{z}^{*}, \lambda_{t}^{*} ; \mu, a\right)$ to only the terms depending on the primal variables $u, z$ and $t$, respectively:

$$
\begin{aligned}
& \mathcal{L}_{u}\left(u, z, t ; \lambda_{z}^{*}, \lambda_{t}^{*} ; \mu, a\right)=\underbrace{\frac{\beta_{z}}{2}\|z-u\|_{2}^{2}+\frac{\beta_{t}}{2}\|t-D u\|_{2}^{2}+\left\langle\lambda_{z}^{*}, u\right\rangle+\left\langle\lambda_{t}^{*}, D u\right\rangle}_{F_{1}(u)}(6.22) \\
& \mathcal{L}_{z}\left(u, z, t ; \lambda_{z}^{*}, \lambda_{t}^{*} ; \mu, a\right)=\underbrace{\frac{\beta_{z}}{2}\|z-u\|_{2}^{2}-\left\langle\lambda_{z}^{*}, z\right\rangle}_{F_{1}(z)}+\underbrace{\frac{\mu}{2}\|z-b\|_{2}^{2}}_{F_{2}(z)} \\
& \mathcal{L}_{t}\left(u, z, t ; \lambda_{z}^{*}, \lambda_{t}^{*} ; \mu, a\right)=\underbrace{-\left\langle\lambda_{t}^{*}, t\right\rangle}_{F_{1}(t)}+\underbrace{\sum_{i=1}^{n} \phi\left(\left\|t_{i}\right\|_{2} ; a\right)+\frac{\beta_{t}}{2}\|t-D u\|_{2}^{2}}_{F_{2}(t)}
\end{aligned}
$$

We notice that the functions $\mathcal{L}_{u}, \mathcal{L}_{z}$ and $\mathcal{L}_{t}$ above are proper, continuous and coercive with respect to the variables $u, z$ and $t$, respectively. Moreover, the three selected functions $F_{1}$ and the function $F_{2}$ in (6.23) are convex, hence $\mathcal{L}_{u}$ and $\mathcal{L}_{z}$ are convex. For what concerns the function $F_{2}$ in (6.24), it follows from the results that are given in Proposition 4.5 that it is strictly convex if and only if the condition $\beta_{t}>a$ is satisfied. Since in the ADMM-based scheme that we will present for solving the saddle-point problem (4.3)-(4.4) such condition will be taken as a constraint, we can assume here that it is satisfied, such that $F_{2}$ in $(6.24)$ is convex and, hence, $\mathcal{L}_{t}$ is convex as well. By finally noticing that the three functions $F_{1}$ are Gâteaux-differentiable, we can apply Lemma 4.3 separately to (6.22), (6.23) and (6.24) thus obtaining the following 
optimality conditions for a generic point $(\bar{u}, \bar{z}, \bar{t})$ :

$$
\begin{aligned}
& \left\langle-\beta_{z}(\bar{z}-\bar{u})-\beta_{t} D^{T}(\bar{t}-D \bar{u})+\lambda_{z}^{*}+D^{T} \lambda_{t}^{*}, u-\bar{u}\right\rangle \geq 0 \quad \forall u \in V, \\
& \frac{\mu}{2}\|z-b\|_{2}^{2}-\frac{\mu}{2}\|\bar{z}-b\|_{2}^{2}+\left\langle\beta_{z}(\bar{z}-\bar{u})-\lambda_{z}^{*}, z-\bar{z}\right\rangle \geq 0 \quad \forall z \in V, \\
& \sum_{i=1}^{n} \phi\left(\left\|t_{i}\right\|_{2} ; a\right)+\frac{\beta_{t}}{2}\|t-D \bar{u}\|_{2}^{2}-\sum_{i=1}^{n} \phi\left(\left\|\bar{t}_{i}\right\|_{2} ; a\right)-\frac{\beta_{t}}{2}\|\bar{t}-D \bar{u}\|_{2}^{2} \\
& -\left\langle\lambda_{t}^{*}, t-\bar{t}\right\rangle \geq 0 \quad \forall t \in Q .
\end{aligned}
$$

We now verify that the triplet $\left(z^{*}, t^{*}, u^{*}\right)$ satisfies the optimality conditions above, so that the second saddle-point condition (6.21) holds true. By substituting $\left(z^{*}, t^{*}, u^{*}\right)$ for $(\bar{z}, \bar{t}, \bar{u})$ in $(6.25),(6.26)$ and $(6.27)$, we obtain:

$$
\begin{aligned}
& \langle-\beta_{z} \underbrace{\left(z^{*}-u^{*}\right)}_{0}-\beta_{t} D^{T} \underbrace{\left(t^{*}-D u^{*}\right)}_{0}+\underbrace{\lambda_{z}^{*}+D^{T} \lambda_{t}^{*}}_{0}, u-u^{*}\rangle \geq 0 \quad \forall u \in V \\
& \frac{\mu}{2}\|z-b\|_{2}^{2}-\frac{\mu}{2}\left\|z^{*}-b\right\|_{2}^{2}+\langle\beta_{z} \underbrace{\left(z^{*}-u^{*}\right)}_{0}-\lambda_{z}^{*}, z-z^{*}\rangle \geq 0 \quad \forall z \in V, \\
& \sum_{i=1}^{n} \phi\left(\left\|t_{i}\right\|_{2} ; a\right)+\frac{\beta_{t}}{2}\left\|t-D u^{*}\right\|_{2}^{2}-\sum_{i=1}^{n} \phi\left(\left\|t_{i}^{*}\right\|_{2} ; a\right)-\frac{\beta_{t}}{2} \underbrace{\left\|t^{*}-D u^{*}\right\|_{2}^{2}}_{0} \\
& -\left\langle\lambda_{t}^{*}, t-t^{*}\right\rangle \geq 0 \quad \forall t \in Q,
\end{aligned}
$$

where the underlined terms are null due to some of the settings in (6.19)-(6.20). The first condition (6.28) is clearly satisfied. We rewrite the second and third condition by substituting also the settings on $\lambda_{z}^{*}$ and $\lambda_{t}^{*}$ in (6.20):

$$
\begin{aligned}
& \frac{\mu}{2}\|z-b\|_{2}^{2}-\frac{\mu}{2}\left\|z^{*}-b\right\|_{2}^{2}-\left\langle\mu\left(z^{*}-b\right), z-z^{*}\right\rangle \geq 0 \quad \forall z \in V \\
& \sum_{i=1}^{n} \phi\left(\left\|t_{i}\right\|_{2} ; a\right)+\frac{\beta_{t}}{2}\left\|t-D u^{*}\right\|_{2}^{2}-\sum_{i=1}^{n} \phi\left(\left\|t_{i}^{*}\right\|_{2} ; a\right)-\frac{\beta_{t}}{2}\left\|t^{*}-D u^{*}\right\|_{2}^{2} \\
& -\left\langle\bar{\partial}_{t}\left[\sum_{i=1}^{n} \phi\left(\left\|t_{i}\right\|_{2} ; a\right)\right]\left(t^{*}\right)+\mu\left(t^{*}-D u^{*}\right), t-t^{*}\right\rangle \geq 0 \quad \forall t \in Q
\end{aligned}
$$

where in $(6.32)$ we added the null term $\mu\left(t^{*}-D u^{*}\right)$. The two optimality conditions (6.31) and (6.32) can be proved based on the concept of Bregman distance, that we thus recall here briefly: given a convex not necessarily smooth function $G$ and two points $x, x^{*}$ in its domain, the Bregman distance (or divergence) associated with function $G$ for points $x, x^{*}$ is defined as

$$
B_{G}\left(x, x^{*}\right):=G(x)-G\left(x^{*}\right)-\left\langle\partial G\left(x^{*}\right), x-x^{*}\right\rangle,
$$

where $\partial G$ denotes the subdifferential of $G$. The Bregman distance is not a distance in strict sense, but it is always non-negative for convex functions $G$. Inequalities (6.31) and (6.32) can be equivalently rewritten in terms of Bregman distances as follows:

$$
\begin{aligned}
& B_{Z}\left(z, z^{*}\right) \geq 0 \forall z \in V, \quad Z(z):=\frac{\mu}{2}\|z-b\|_{2}^{2}, \\
& B_{T}\left(t, t^{*}\right) \geq 0 \quad \forall t \in Q, \quad T(t):=\sum_{i=1}^{n} \phi\left(\left\|t_{i}\right\|_{2} ; a\right)+\frac{\beta_{t}}{2}\left\|t-D u^{*}\right\|_{2}^{2} .
\end{aligned}
$$


In particular, (6.34) follows immediately from (6.31) and (6.33) whereas (6.35) follows from (6.32) and (6.33) and from two further observations. First, the function $T$ in (6.35) is convex for the same reasons for which the function $F_{2}$ in (6.24) is convex. Second, the first term of the scalar product in (6.35) represents the subdifferential of the convex function $T$. Since the Bregman distance is always non-negative, (6.34) and (6.35) hold true, the second saddle-point condition in (6.21) is satisfied and, finally, the second part of the Theorem proof is completed.

$\square$

\section{Proof of Proposition 4.5.}

Proof. Condition (4.32) for convexity of function $\theta$ in (4.31) can be easily demonstrated based on Proposition 3.3 in Section 3. In fact, after rewriting $\theta$ as follows:

$$
\theta(x)=\phi\left(\|x\|_{2} ; a\right)+\frac{\beta}{2}\|x\|_{2}^{2}+L(x), \quad x \in \mathbb{R}^{2},
$$

with $L$ an affine function which, hence, does not affect convexity, we notice that the non-affine part of $\theta$ in (6.36) can be written in composite form as

$$
h(\rho(x)), \text { with } \rho(x):=\|x\|_{2}, x \in \mathbb{R}^{2} \text { and } h(t):=\phi(t ; a)+\frac{\beta}{2} t^{2}, t \geq 0 .
$$

Hence, recalling demonstration of Proposition 3.3, the function $\theta$ is convex if and only if the following two conditions both hold:

$$
\left\{\begin{array} { l } 
{ h ^ { \prime } ( t ) > 0 } \\
{ h ^ { \prime \prime } ( t ) > 0 }
\end{array} \equiv \left\{\begin{array} { l } 
{ \phi ^ { \prime } ( t ; a ) + \beta t > 0 } \\
{ \phi ^ { \prime \prime } ( t ; a ) + \beta > 0 }
\end{array} \equiv \left\{\begin{array}{l}
\beta t>-\phi^{\prime}(t ; a) \\
\beta>-\phi^{\prime \prime}(t ; a)
\end{array} \quad \forall t \geq 0 .\right.\right.\right.
$$

Since by hypothesis $\beta>0$ and the function $\phi$ satisfies assumption A2) in Section 2, namely $\phi^{\prime}(t ; a)>0$ for any $t \geq 0$, the first condition in (6.38) is always satisfied. The second condition in (6.38) is equivalent to the convexity condition in statement (4.32).

We now prove statement (4.34), according to which the unique solution $x^{*}$ of the strictly convex problem (4.33) is obtained by a shrinkage of vector $r$. To allow for a clearer understanding of the proof, in Figure 6.1 we give a geometric representation of problem (4.33). First, we prove that the solution $x^{*}$ of (4.33) lies on the closed half-line $O r$ with origin at the 2-dimensional null vector $O$ and passing through $r$, represented in solid red in Figure 6.1(a). To this purpose, we demonstrate that for any point $z$ not lying on $O r$ there always exists a point $z^{*}$ on $O r$ providing a lower value of the objective function in (4.33), that is a point $z^{*}$ such that $\theta(z)-\theta\left(z^{*}\right)>0$. In particular, we define $z^{*}$ as the intersection point between the half-line $O r$ and the sphere with center in $O$ and passing through $z$, depicted in solid blue in Figure 6.1(a). Recalling the definition of $\theta$ in (4.31) and noting that $\left\|z^{*}\right\|_{2}=\|z\|_{2}$ by construction, we can thus write:

$$
\begin{aligned}
\theta(z)-\theta\left(z^{*}\right) & =\phi\left(\|z\|_{2} ; a\right)-\phi\left(\left\|z^{*}\right\|_{2} ; a\right)+\frac{\beta}{2}\left(\|z-r\|_{2}^{2}-\left\|z^{*}-r\right\|_{2}^{2}\right) \\
& =\frac{\beta}{2}\left(\|z\|_{2}^{2}+\|r\|_{2}^{2}-2\langle z, r\rangle-\left\|z^{*}\right\|_{2}^{2}-\|r\|_{2}^{2}+2\left\langle z^{*}, r\right\rangle\right) \\
& =\beta\left\langle z^{*}-z, r\right\rangle \\
& =\beta\left\|z^{*}-z\right\|_{2}\|r\|_{2} \cos \left(\widehat{O z^{*} z}\right) .
\end{aligned}
$$




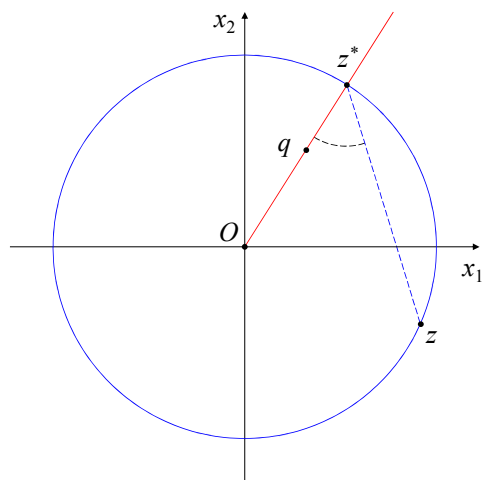

(a)

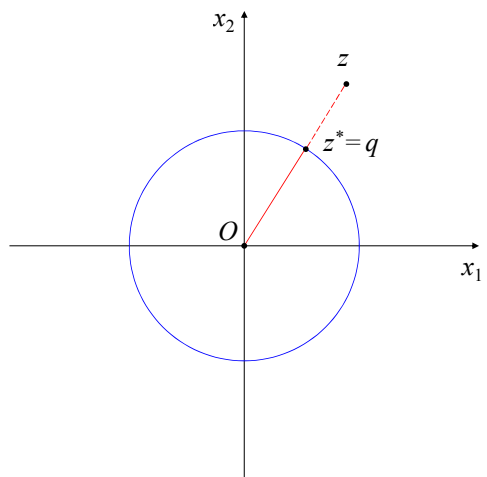

(b)

FiG. 6.1. Geometric representation of problem (4.33).

Since $\beta>0$ by hypothesis, $z^{*} \neq z$ and $r \neq O$ by construction, and noting that the angle $\widehat{O z^{*} z}$ is always acute, we can conclude that the expression in (6.39) is positive. Hence, the solution $x^{*}$ of (4.33) lies on the closed half-line $O r$.

We now prove that the solution $x^{*}$ of (4.33) lies inside the segment $[O r]$, represented in solid red in Figure 6.1(b). To this purpose, we demonstrate that for any point $z$ lying on the half-line $O r$ but outside the segment $[\mathrm{Or}]$ there always exists a point $z^{*}$ on $[O r]$ such that $\theta(z)-\theta\left(z^{*}\right)>0$. In particular, it suffices to choose $z^{*}=r$, as illustrated in Figure 6.1(b). We obtain:

$$
\theta(z)-\theta\left(z^{*}\right)=\theta(z)-\theta(r)=\phi\left(\|z\|_{2} ; a\right)-\phi\left(\|r\|_{2} ; a\right)+\frac{\beta}{2}\|z-r\|_{2}^{2} .
$$

Since $\|z\|_{2}>\|r\|_{2}$ by construction and the function $\phi$ is monotonically increasing by hypothesis, the expression in (6.40) is positive, hence the solution $x^{*}$ of (4.33) lies on the segment $[O r]$.

To conclude the proof of statement (4.34), we notice that the directional derivative of the objective function $\theta$ in (4.31) at $r$ in the direction of $r$ is as follows:

$$
\frac{\partial \theta}{\partial r}(r)=\phi^{\prime}\left(\|r\|_{2} ; a\right)>0,
$$

where the inequality follows from assumption A2) in Section 2. It follows from (6.41) that the solution $x^{*}$ of (4.33) never coincides with vector $r$.

Based on (4.34), by setting $x=\xi r, 0 \leq \xi<1$, the original unconstrained 2-dimensional problem in (4.33) can be reduced to the following equivalent constrained 1-dimensional problem:

$$
\begin{aligned}
\xi^{*} & \leftarrow \arg \min _{0 \leq \xi<1}\left\{\phi\left(\|\xi r\|_{2} ; a\right)+\frac{\beta}{2}\|\xi r-r\|_{2}^{2}\right\} \\
& \leftarrow \arg \min _{0 \leq \xi<1}\left\{f(\xi)=\phi\left(\|r\|_{2} \xi ; a\right)+\frac{\beta}{2}\|r\|_{2}^{2} \xi(\xi-2)\right\},
\end{aligned}
$$

where in (6.42) we omitted the constant terms and introduced the objective function $f$ for future reference. Since we are assuming that the function $\phi$ is twice continuously differentiable in $\mathbb{R}_{+}$, so it is the cost function $f$ in (6.42) in the optimization domain 
$0 \leq \xi \leq 1$. Moreover, $f$ is strictly convex since it represents the restriction of the strictly convex function $\theta$ in (4.31) to the segment $\xi r, 0 \leq \xi \leq 1$. Hence, the necessary and sufficient condition for an inner point $0<\xi<1$ to be the global minimizer of $f$ is as follows:

$$
f^{\prime}(\xi)=0 \equiv \Downarrow r H_{2}\left[\phi^{\prime}\left(\|r\|_{2} \xi ; a\right)+\beta\|r\|_{2}(\xi-1)\right]=0 .
$$

Since $f$ is strictly convex, the first derivative $f^{\prime}(\xi)$ is strictly increasing in the entire domain $0 \leq \xi \leq 1$ and at the extremes we have:

$$
f^{\prime}\left(0^{+}\right)=\|r\|_{2}\left[\phi^{\prime}\left(0^{+} ; a\right)-\beta\|r\|_{2}\right], \quad f^{\prime}(1)=\|r\|_{2} \phi^{\prime}\left(\|r\|_{2} ; a\right) .
$$

Since $\|r\|_{2}>0$ and $\phi^{\prime}(t ; a)>0$ for any $t \geq 0$ by hypothesis, $f^{\prime}(1)$ in (6.44) is positive. Hence, we have two cases. If $f^{\prime}\left(0^{+}\right) \geq 0$, that is $\|r\| \leq \phi^{\prime}\left(0^{+} ; a\right) / \beta, f^{\prime}(t)$ is positive in $0<t \leq 1$, hence the function $f$ has its minimum at $\xi^{*}=0$; if $f^{\prime}\left(0^{+}\right)<0$, then $f$ has the minimum at its unique stationary point $0<\xi^{*}<1$, which can be obtained by solving the nonlinear equation in (6.43).

\section{Proof of Proposition 4.6.}

Proof. After setting $\alpha:=\|r\|_{2}$ for simplicity of notations, we have to solve the following constrained nonlinear equation:

$$
\phi^{\prime}(\alpha x ; a)+\beta \alpha(x-1)=0, \quad 0<x<1, \quad \alpha \beta>1, \quad \beta>a .
$$

Substituting in (6.45) the expression of the first-order derivative of the exponential penalty function reported in the second row of Table 2.1, we obtain:

$$
\frac{1}{e^{a \alpha x}}+\beta \alpha(x-1)=0 \equiv 1+\beta \alpha(x-1) e^{a \alpha x}=0 \equiv \beta \alpha(x-1) e^{a \alpha x}=-1
$$

We notice that:

$$
e^{a \alpha x}=e^{a \alpha} e^{a \alpha(x-1)}
$$

such that (6.46) can be written as:

$$
\beta \alpha(x-1) e^{a \alpha} e^{a \alpha(x-1)}=-1 .
$$

After multiplying both sides of (6.48) by $a /\left(\beta e^{a \alpha}\right)$, we obtain:

$$
a \alpha(x-1) e^{a \alpha(x-1)}=-\frac{a}{\beta e^{a \alpha}} .
$$

After the following change of variable:

$$
y=a \alpha(x-1)
$$

we obtain:

$$
\left.y e^{y}=-\frac{a}{\beta e^{a \alpha}}, \quad y \in\right]-a \alpha, 0[,
$$

Hence, the unique solution $y^{*}$ of $(6.51)$ is given by:

$$
y^{*}=W_{0}\left(-\frac{a}{\beta e^{a \alpha}}\right),
$$

and, following from (6.50), the unique solution $x^{*}$ of (6.46) is:

$$
x^{*}=1+\frac{1}{a \alpha} W_{0}\left(-\frac{a}{\beta e^{a \alpha}}\right),
$$

where $W_{0}(\cdot)$ represents the principal branch of the Lambert $\mathrm{W}$ function [10]. 